\title{
In situ Neutron Diffraction Evidence For Fully Reversible Dislocation Motion in Highly Textured Polycrystalline $\mathrm{Ti}_{2} \mathrm{AlC}$ Samples
}

\author{
Mohamed Shamma, ${ }^{1}+$ El'ad N. Caspi, ${ }^{1, \#}+$, Babak Anasori, ${ }^{1}$ Bjørn Clausen, ${ }^{3}$ Donald W. Brown, \\ Sven C. Vogel, ${ }^{3}$ Volker Presser, ${ }^{1,2}$ Shahram Amini, ${ }^{1}$ Ori Yeheskel ${ }^{1,5}$ and Michel W. Barsoum ${ }^{1, *}$ \\ ${ }^{1}$ Department of Materials Science and Engineering, Drexel University, Philadelphia, Pennsylvania 19104, USA \\ ${ }^{2}$ A. J. Drexel Nanotechnology Institute, Drexel University, Philadelphia, Pennsylvania 19104, USA \\ ${ }^{3}$ Lujan Center, Los Alamos National Laboratory, Los Alamos, New Mexico 87545, USA \\ ${ }^{4}$ MST-8, Los Alamos National Laboratory, Los Alamos, New Mexico 87545, USA \\ ${ }^{5}$ Nuclear Research Center-Negev, P.O. Box 9001, Beer Sheva 84190, Israel \\ \# - On sabbatical leave from the Nuclear Research Centre - Negev, Israel \\ $\ddagger$ Contributed equally to this work
}

- -Corresponding author. Email: barsoumw@drexel.edu

Herein careful analysis of in situ neutron diffraction patterns obtained, while cyclically loading highly textured polycrystalline $\mathrm{Ti}_{2} \mathrm{AIC}$, a MAX phase, samples, provides compelling experimental evidence - in the form of fully reversible peak lattice elastic strain loops and peak widening and narrowing upon load cycling - for the existence of fully reversible dislocation motion. A comparison of the measured and calculated dislocation densities clearly shows that dislocation pileups alone cannot account for the experimental observations. Another micromechanism needs to be invoked. Based on the propensity of the MAX phases to deform by kinking, the micromechanism proposed is either the nucleation and annihilation of incipient kink bands, IKB, and/or the bowing of dislocations in preexisting low angle kink boundaries, LAKB. This micromechanism plays a vital role during the initial deformation of layered and other plastically anisotropic solids such as hexagonal close-packed metals. Consequently, the ramifications of this work on geology, metallurgy and other fields will prove quite important.

\section{Introduction}

In 1934, Orowan, Polanyi and Taylor, roughly simultaneously, realized that plastic deformation could be explained in terms of the theory of dislocations. Since that time, dislocation slip, together with twinning, have been the two fundamental micromechanisms invoked to understand plastic deformation of most solids. In 2003 we observed fully and spontaneously reversible stress-strain loops when $\mathrm{Ti}_{3} \mathrm{SiC}_{2}$ cylinders were cyclically compressively loaded. At $1 \mathrm{GPa}$, the energy dissipated per cycle was roughly $25 \%$ of the total energy.[1] We labeled this phenomenon kinking nonlinear elasticity or KNE: Elastic because the process was fully and spontaneously reversible, nonlinear because the stress-strain curves were nonlinear (Figs. 1(a) and b) and kinking to emphasize that dislocations in the form of kink boundaries were involved. 
We conjectured that the micromechanism responsible for KNE behavior was the incipient kink band or IKB. The latter is comprised of multiple, co-axial parallel dislocation loops (inset in Fig. 1(a)) that only extend under stress; when the stress is removed they disappear. It was also postulated that it is the to-and-fro motion of these dislocations, in turn, dissipate the energy labeled $\mathrm{W}_{\mathrm{d}}$ in Fig. 1(a). Over the past decade we have shown that KNE solids are quite ubiquitous and exist in many types of solids: from graphite to mica, to hexagonal metals, to the MAX phases (see below).[2] In 2005, we developed a micromechanical model [3] to explain KNE behavior and have since published several papers in which we presented strong circumstantial evidence for the existence of IKBs.[4-7]

IKBs are elusive because they only appear under load and disappear when the load is removed. Furthermore, the concomitant shear is quite small; in most cases, and as shown here, less than a degree. Therefore, an in situ approach is required. Herein we used in situ stress-strain neutron diffraction, ND, measurements, together with the Elasto-Plastic Self-Consistent (EPSC) modeling approach [8-12] to shed light on the deformation of highly textured $\mathrm{Ti}_{2} \mathrm{AlC}$ samples. The advantage of in situ ND measurements is the method's ability to probe the bulk's microscopic structural elastic response, while the EPSC analysis approach connects the elastic and plastic properties with the texture of a polycrystalline material at both the bulk and individual reflections levels.

The paper deals with the ternary carbide, $\mathrm{Ti}_{2} \mathrm{AlC}$, a member of the MAX phases. The latter are a large family ( $>70$ members) of ternary early transition metal carbides, carbonitrides, and nitrides with a layered hexagonal structure and a $\mathrm{M}_{n+1} \mathrm{AX}_{n}$ chemistry, where: " $\mathrm{M}$ " is an early transition metal, "A" is an A-group element (mostly groups 13 and 14), " $\mathrm{X}$ " is carbon or nitrogen, and $n=1,2,3$, etc.[13] What sets these phases apart is, as noted above, their unusual response to stress. Upon cyclic loading, these phases trace fully and spontaneously reversible, strain rate-independent, closed hysteresis loops.[7, 14] The magnitudes of $\mathrm{W}_{\mathrm{d}}$ depend on grain size $[4,7]$ and, as shown herein, also on texture.

The ultimate purpose of our work in this area is to understand the underlying mechanisms that result in non-linear elastic behavior and the origin of the concomitant energy dissipation. As shown herein classic plasticity mechanisms such as dislocation pileups, DPs, and/or twinning, cannot explain some key observations in the mechanical behavior of the MAX phases, especially in the low strain regime. This paper is an important step towards this goal.

Herein we compressively load two relatively coarse-grained (Fig. 2), textured, $\mathrm{Ti}_{2} \mathrm{AlC}$ polycrystalline cylinders, while in situ measuring their microscopic crystallographic response using ND. Up to at least $1200{ }^{\circ} \mathrm{C}$, the MAX phases possess no more than two independent slip systems, viz. basal dislocations.[15, 16] Twins have never been reported and/or implicated in the deformation and are not considered hereafter. The MAX phases are thus hugely plastically anisotropic and possess less than the 5 independent slip systems needed for general ductility. When in polycrystalline form, such plastically anisotropic materials respond to stress by either microcracking and/or by developing residual elastic stresses.

Another consequence of this plastic anisotropy is the differentiation of grains into "soft" and "hard" grains. Grains oriented with their basal planes either parallel or normal to the loading direction, will have negligible Schmidt factors, and are considered hard grains. Deviation from these two extreme orientations, "softens" the grains to shear, with the softest grains having their 
basal planes oriented at $\approx 45^{\circ}$ to the applied load. As shown below, the hard and soft grains play complementary roles in the overall deformation of our textured samples.

Recently, Guitton et al.[17] carried out neutron diffraction measurements with in-situ compression tests together with "post-mortem" SEM observations to study the stress-strain behavior of a non-textured polycrystalline $\mathrm{Ti}_{2} \mathrm{AlN}$ sample. The main result of their work suggests the existence of a combination of soft and hard grains in the polycrystal, in agreement with our observations. As we show here, they too observe that upon loading the soft grains reach their plastic regime, while the hard grains retain their elastic behavior. Guitton et al., however, conclude that their observations can be described using the classical elasto-plastic mechanism applied to a complex and highly geometrical anisotropic polycrystalline system, which causes a complex and highly anisotropic stress field upon loading. Said otherwise, they conclude that DPs in the soft grains can explain the totality of their results and there is no need to invoke other mechanisms such as kink boundaries for example. The same conclusion was reached by Jones et al. [18] In the latter case, however, the analysis leaves a lot to be desired for many reasons the most glaring being the use of quite noisy (0002) peaks instead of the much more intense (0008) peaks to make their case.

This paper is structured as follows. First we simply show the results and make the case that while some reflections are behaving as one would expect them to behave in highly plastically anisotropic solids, crucially other important reflections from some of the hardest grains quite simply are "not playing by the rules". In the second part we show that some reflections from these hard grains broaden, and narrow, fully reversibly and spontaneously with cycling, providing unambiguous evidence for a fully reversible increase/decrease in dislocation density with cycling. In that section it is also shown that the changes in dislocation density are incompatible with DPs alone and that another micromechanism needs to be invoked. In the third part, the EPSC model is implemented in an attempt to make sense of the changes in the microscopic strains. This exercise, in turn, strongly supports our conjecture that another micromechanism in addition to DPs is indeed needed to explain the results. We then make the case why this micromechanism is either $2 \mathrm{D}$ IKBs or the bowing of dislocations in pre-existing low angle kink boundaries, before concluding by highlighting the importance of this work and its implications.

\section{Experimental details}

\section{Materials}

Highly textured samples were fabricated by hot pressing, HPing, pre-reacted $\mathrm{Ti}_{2} \mathrm{AlC}$ powders (Kanthal Corp. Sweden) at $1500{ }^{\circ} \mathrm{C}$ for $4 \mathrm{~h}$. Sample texture was achieved by mechanically tapping the plate-like powders (aspect ratio $\approx 5$ ) before hot pressing.

Two cylinders (30 $\mathrm{mm}$ high, $9 \mathrm{~mm}$ diameter) were machined - from the same HPed billet - by electrical discharge machining. One sample, henceforth referred to as "N", was machined such that the basal planes were normal to the loading axis (inset (i) in Fig. 1(b)). The other sample, referred to herein as "P", was machined such that the basal planes were loaded edge-on (inset (ii) in Fig. 1(b)). In these schematics the fine lines denote basal planes and the rectangles represent grains. In both samples the majority of the grains (represented by black 
rectangles) orient in a hard direction. The red rectangles represent minority grains also oriented in a hard direction; the blue ones represent grains oriented in a soft direction.

\section{Methods}

The samples' textures were determined using the High Pressure Preferred Orientation (HIPPO) neutron diffractometer,[19] at the Lujan Neutron Scattering Center (LANSCE), Los Alamos National Laboratory (LANL). Texture analysis was performed using the Rietveld refinement method with the Material Analysis Using Diffraction (MAUD) code, utilizing the Williams-Imhof-Matthies-Vinel (WIMV) method.[20]

In situ compression and ND measurements were undertaken using the Spectrometer for Materials Research at Temperature and Stress (SMARTS) instrument at LANSCE, LANL.[21] The relationship between the neutron beam, detectors (Bank 1 - Transverse; Bank 2 Longitudinal), and loading direction in SMARTS, is shown schematically in Fig. 1(c).

According to the law of diffraction the scattering vector of a grain is parallel to the direction formed by the vectoric difference of the final beam $\left(\mathbf{k}_{\mathbf{f}}\right)$ and initial beam $\left(\mathbf{k}_{\mathbf{i}}\right)$ directions, i.e. $\mathbf{k}_{\mathbf{s}}=\mathbf{k}_{\mathbf{f}}-\mathbf{k}_{\mathbf{i}}$. It follows that, in the diffraction configuration in this work (Fig. 1(c)), the diffraction pattern collected with the longitudinal (transverse) detectors corresponds to grains with scattering vectors which are parallel (perpendicular) to the compression direction. As an example, "black" grains in Fig. 1(b) (inset (i)) are oriented in a way that the scattering vector of the longitudinal direction is parallel to the normal to the basal planes (i.e. $\{000 l\}$ ), and therefore, diffraction from these grains to the longitudinal detectors should correspond directly to the stress applied along the hexagonal c axis.

It is important to note that essentially the same volume of the same sample was probed by both HIPPO and SMARTS. The latter were taken at 10 loading points for the N-orientation, and 19 for the $\mathrm{P}$, with the initial and final points at a nominal compressive load of $5 \mathrm{MPa}$ (needed to keep the sample in place). The bulk stress-strain behavior (Fig. 1(b)) was recorded continuously and simultaneously to the ND measurements, using an extensometer directly attached to the samples. The ND data were analyzed by both the Rietveld refinement method, using the General Structure Analysis System (GSAS) with its Graphic User Interface (EXPGUI) code (Fig. 3) [22, 23] and single peaks fit using the RAWPLOT subroutine embedded in GSAS, scripted for batch refinements using SMARTSware [24].

Stress vs. microstrain curves, obtained for each analyzed ND peak, were compared to those generated from the EPSC calculations. The EPSC approach treats grains as inclusions in an infinite homogeneous matrix whose properties are equal to the average polycrystal moduli computed from the single crystal moduli for all other grains in the model, taking into account their orientation and shape. The EPSC approach incorporates elastic and plastic anisotropy,[9] but does not incorporate KBs.

\section{Results}

\section{Sample Characteristics}

Figure 2 shows an optical microscope, OM, micrograph of a polished and etched cross- 
section of the $\mathrm{Ti}_{2} \mathrm{AlC}$ sample tested herein. The samples were fully dense $\left(4.1 \mathrm{~g} / \mathrm{cm}^{3}\right)$, with a final average grain diameter, $\lambda$, of $60 \pm 40 \mu \mathrm{m}$ and a thickness, $2 \alpha$, of $25 \pm 10 \mu \mathrm{m}$ (Fig. 2).

\section{Neutron Diffraction}

In both the $\mathrm{N}$ - and P-samples three MAX phases were observed in both HIPPO and SMARTS. Rietveld analysis of the data sets is consistent with the existence of $71 \pm 5 \mathrm{wt} \%$, $16 \pm 3$ wt. $\%$ and $13 \pm 4$ wt. $\%$ of the $\mathrm{Ti}_{2} \mathrm{AlC}, \mathrm{Ti}_{3} \mathrm{AlC}_{2}$, and $\mathrm{Ti}_{5} \mathrm{Al}_{2} \mathrm{C}_{3}$ phases, respectively. The TiC content was $\approx 1 \mathrm{wt} \%$. In both samples, all three phases had similar majority/minority orientation textures. Excellent Rietveld fits with goodness of fit of $\chi^{2}<2.5$, and $\mathrm{R}_{\mathrm{wp}} \leq 4.5 \%$ for all SMARTS data (e.g. Fig. 3), full identification of all observed diffraction lines, as well as the existence of substantial number of isolated $\mathrm{Ti}_{2} \mathrm{AlC}$ peaks in the diffraction patterns, led to the results presented herein with almost negligible systematic errors, impurity phases notwithstanding. The structure and texture similarities of the three observed phases, as well as the negligible experimental systematic error in the ND analysis, enables us to confidently consider the $\mathrm{Ti}_{2} \mathrm{AlC}$ phase solely when comparing the ND results to the single phase EPSC calculation. This is especially true here since the impurity phases are also MAX phases with quite similar elastic and plastic properties as the $\mathrm{Ti}_{2} \mathrm{AlC}$ majority phase. All other fits (not shown) were equally good.

Analysis of the ND data for texture determination, collected on HIPPO, revealed significant texture consistent with the predicted basal planes' orientations (compare Figs. 4(a) $\& 4(\mathrm{c}))$. The $\mathrm{N}$-sample showed a maximal value of $\approx 6.1$ random; the P-sample $\approx 4.6$ times. In other words, the $\mathrm{N}$-sample was more textured than the P-sample. As expected from the experimentally observed texture, a minor, yet significant amount of differently oriented grains is also observed in both samples, as exemplified by the pole plots for the 10-15 reflection planes of the $\mathrm{N}$ - and P-samples shown in Figs. 4(b) \& 4d, respectively. These results support the samples' configurations shown as schematics in the insets of Fig. 2(b).

\section{Macroscopic Stress-Strain Curves}

Figure 1(b) plots the bulk stress-strain curves for the N- and P-samples, represented by the blue and red loops, respectively. In this and all similar figures, both stress and strain axes are reversed to render the plots similar to conventional tensile stress-strain curves. From these results it is obvious that the $\mathrm{N}$-sample is less rigid and dissipates more energy per unit volume per cycle.

\section{Microscopic Stress-Strain curves}

Microscopic stress-strain curves, determined from single peak fits of the $\{0001\},\{10 \overline{1} 0\}$ and various $\{10 \overline{1} l\}$ reflections in the ND data along the longitudinal and transverse directions to the compressive loading axis, for the $\mathrm{N}$ - and P-samples are shown in Figs. 5 and 6. The experimental results on loading are depicted by solid symbols; those during unloading by open ones. The EPSC model results - assuming linear elastic behavior only - are depicted in all figures by dashed green lines. The EPSC model results - assuming plastic deformation by basal slip - are depicted in Fig. 6 by dotted red lines.

\section{Discussion}


As noted above, due to their high c/a ratios (3 to 8), neither non-basal dislocations nor twins have ever been implicated in the deformation of the MAX phases under normal loading conditions. Referring to insets (i) and (ii) in Fig. 1(b), it is therefore clear that in both microstructures the majority of grains - represented by the black grains - are hard. The blue colored grains, on the other hand, are soft. For example, in $\mathrm{Ti}_{2} \mathrm{AlC}$, the angle between the basal planes and the normal to the $\{10 \overline{1} 5\}$ reflections is $\sim 42^{\circ}$. It follows that these grains are the ones for which the resolved shear stresses, RSSs, are the highest and therefore are the ones that, as shown herein, deform by slip, where the dislocations are presumably arranged in the form of dislocation pileups, DPs.

Before proceeding much further, it is instructive to briefly summarize how the ND signatures of soft and hard grains - in plastically anisotropic solids - differ. In all cases, the initial response is linear elastic. At the yield point, the soft grains deform and shed their load unto the hard grains, which results in a curling upward of the elastic strains of the soft grains and a curling toward the horizontal of the hard grains. This response is true for both the longitudinal and transverse directions. Figures 5(a) and (b) plot the elastic strains obtained from single peak fits of various peaks indicated for the $\mathrm{N}$-sample in the transverse and longitudinal direction, respectively. Figures 5(c) and (d) plot the corresponding curves for the P-sample. A perusal of these results quickly establishes that, with the notable exception of the hardest grains discussed separately below, all others reflections play by the rules. For example, the $\{10 \overline{15}\}$ plane reflections are from grains whose basal planes make an angle of $\approx 42^{\circ}$ with that of the applied load. They thus originate from some of the softest grains and indeed show clear signs of plastic deformation in both the $\mathrm{N}$ - and P-samples (see blue curves and data points in Fig. 5). The $\{10 \overline{1} 1\}$ reflections, however, are from grains whose basal planes make an angle of $\approx 13^{\circ}$ with that of the applied load. They thus originate from hard grains and it is thus not surprising that in this case, the elastic strains are displaced in the opposite direction of the soft grains (see orange curves and data points in Fig. 5). The other reflections are in between these two extremes.

We now turn our attention to the $\{0001\}$ and $\{10 \overline{1} 0\}$ reflections that originate in the hardest of grains since the RSS on their basal planes is quite low; in a perfectly aligned sample it is vanishingly low. In this case, common sense predicates that these planes deflect farthest from a linear elastic response. Figure 6 plots the response of these reflections from which it is clear that the elastic strains in these grains are almost linear elastic. This is a crucial observation since it unambiguously signifies that these grains are significantly "softer" than one would have otherwise anticipated. Said otherwise, due to their negligible RSS, these grains should have accommodated or shouldered the loads shed by the softer grains and thus should have experienced the largest elastic strains. However, the results show that they yield, but not in the way expected by DPs. This is a crucial and critical observation that we submit is the signature of fully reversible dislocation motion, that originates from either the nucleation and annihilation of incipient kink bands, IKBs, and/or the bowing (and then release) of dislocations in preexisting low angle kink boundaries (LAKB).

This last point is worth emphasizing since it represents one of the main findings of this work. When the single peak fits of the (0004) reflections in the longitudinal direction (solid blue data points and lines in Fig. 7) are plotted, they respond in an almost linear elastic fashion depicted by the inclined green dotted line. These basal planes are thus carrying a significantly lower portion of the stress they would have carried had they deformed by DPs given by the 
dotted black lines (see below). Said otherwise, instead of carrying a stress that would cause them to deform to an elastic strain of $2300 \times 10^{-6}$ (point $X$ in Fig. 7 ), in reality they only deformed to a stress corresponding to an elastic strain of $1750 \times 10^{-6}$ (i.e. to point $Y$ in Fig. 7).

Some may argue that these planes are simply responding in a linear elastic fashion because they are quite hard and thus cannot deform. This argument, however, is untenable for the simple reason that the response is not linear elastic since energy is dissipated in each cycle. The stress strain curves shown in Fig. 7 trace fully and spontaneously reversible, clockwise loops (Fig. 7). The same arguments can be made for the (0004) reflections in the transverse direction for the P-sample (solid red data points and lines in Fig. 7). This clearly rules out linear elasticity. It is important to note that the error bars in Fig. 7 are smaller than the size of the symbols and thus outside the noise.

A perusal of the $\{10 \overline{1} 0\}$ reflections in Fig. 6 clearly shows that the same is occurring with these hard planes: Here again the response is almost linear elastic and dissipative.

\section{Dislocation Densities}

Probably the strongest evidence that something "unusual" is occurring in the hardest grains is to plot the full width at half-maximum, FWHM, of the $(000 l)$ reflections as a function of applied stress. Figure 8 plots the (0004) reflections for the N-sample, from which it is clear that a small, yet statistically significant, broadening of this peak occurs. Most remarkably, this peak broadening is fully and spontaneously reversible. To the best of our knowledge, such fully reversible elastic microstrain hysteresis loops (Figs. 7, and 8) are the first of their kind to be reported. Such reversible broadening is also expected upon loading for the $\{10 \overline{1} 0\}$ reflections in the P-sample. However, the lower intensity of this reflection compared to that of the (0004) reflections in the $\mathrm{N}$-sample, along with the fact that this effect is roughly twice as small as the effect in the latter (Fig. 1(b)), renders the widths variations of these peaks quite noisy and inconclusive (see inset in Fig. 8).

Before estimating the dislocation densities, it is important to define some terms. The total strain, $\varepsilon_{\text {tot }}$, of a solid loaded to an uniaxial stress, $\sigma$, is given by:

$$
\varepsilon_{t o t}=\varepsilon_{E L}+\varepsilon_{N L}=\frac{\sigma}{E}+\left[\varepsilon_{K B}+\varepsilon_{D P}\right]
$$

where $\mathrm{E}$ is Young's modulus, $\varepsilon_{\mathrm{EL}}$ is the elastic strain and $\varepsilon_{\mathrm{NL}}$ is the total nonlinear strain shown schematically in Fig. 1(a). The strains $\varepsilon_{\mathrm{KB}}$ and $\varepsilon_{\mathrm{DP}}$ - both fully reversible - are associated with the kink bands and DPs, respectively.

Frank and Stroh, F\&S, [25] considered a 2 dimensional, 2D, elliptic kink band, KB, (with length, $2 \alpha$, and width, $2 \beta$, such that $\alpha>>\beta-$ see Fig. 1(d)) and showed that the critical angle of an IKB, $\gamma_{c}$, is given by:

$$
\gamma_{c}=\frac{b}{D} \approx \frac{3 \sqrt{3}(1-v) \tau_{l o c}}{2 G} \approx \frac{3 \sqrt{3}(1-v)}{8 \pi e}\left(\frac{b}{w}\right)
$$

where $G$ is the shear modulus and $v$ is Poisson's ratio and $\tau_{\text {loc }}$ is the local shear stress needed to nucleate a dislocation pair; $\mathrm{b}$ is the Burgers vector, $\mathrm{D}$ is the distance between dislocation loops 
along $2 \alpha$ (inset in Fig. 1(a) and 1(d)), w is the dislocation core width. If one assumes, $w=b$, then $\tau_{\text {loc }} \approx \mathrm{G} / 30$, and $\gamma_{\mathrm{c}}$ is of the order of $0.05 \mathrm{rad}$. If, as assumed here and in our previous work[4-7], $\mathrm{w}=5 \mathrm{~b}$, then $\gamma_{\mathrm{c}}=0.012 \mathrm{rad}$.

The dislocation density, $\rho_{\exp }$, can be calculated from the broadening, $\Delta \mathrm{d} / \mathrm{d}_{0}$ of the various diffraction peaks. The two are related by:[26]

$$
\rho_{\text {exp }}=\frac{3 E \phi}{G^{2}\left(1+2 v^{2}\right)}\left(\frac{\Delta d}{d}\right)^{2}
$$

where $\phi$ is a factor that takes into account how the dislocations are interacting. As discussed in Appendix A, this factor is assumed to be between 1 for an IKB with $\gamma_{c}=0.012$ rad and $\approx 9$ for a $0.09 \mathrm{rad}$, or a $5^{\circ}, \mathrm{LAKB}$. For the DP we, assume that, like Ref. [25], their energies are equal to non-interacting dislocations and thus $\phi=1$.

At maximum stress, the measured $\Delta \mathrm{d} / \mathrm{d}_{0}$ was $\sim 3.4 \times 10^{-4}$ (Fig. 8). Assuming $\mathrm{b}=0.306$ $\mathrm{nm}, \mathrm{E}=277 \mathrm{GPa}, \mathrm{G}=118 \mathrm{GPa}$, and $v=0.19$, [4], from Eq. 3, $\rho_{\text {exp }}$ is calculated to be $8(2) \times 10^{12}$ $\mathrm{m}^{-2}$ for IKB or DP $(\phi=1)$, and $7(2) \times 10^{13} \mathrm{~m}^{-2}$ for LAKB $(\phi=9)$. The estimated uncertainty is mainly due to the uncertainty in measured $\Delta \mathrm{d} / \mathrm{d}_{0}$.

The next step is to compare this value to that of various micromechanisms that can cause such a reversible increase in dislocation densities. Here we consider three possibilities: dislocation pileups, $\rho_{\mathrm{DP}}$, the $2 \mathrm{D}$ IKBs postulated by $\mathrm{F} \& S, \rho_{2 \mathrm{D}}$ [25], and the prolate spheroids, whose existence we postulated, $\rho_{\text {IKB }}$ (inset in Fig. 1(a)).[3]

The corresponding dislocation densities are given by (Appendix B):

$$
\rho_{D P} \approx \frac{2.1(1+v) \varepsilon_{D P}}{M b \lambda}
$$

where $\mathrm{M}$ is the Taylor factor. For the prolate spheroids (Appendix B),

$$
\rho_{I K B} \approx \frac{3 k_{1} \varepsilon_{I K B}}{2 b \beta_{a v}}
$$

where $\mathrm{k}_{1}$ relates the IKB's volumetric strain to the axial strain measured along the loading direction. Similarly, if the IKB shape is that shown in Fig. 1(d), then,

$$
\rho_{2 D} \approx \frac{4 k_{2} \varepsilon_{2 D}}{\pi b \beta}
$$

here again $\mathrm{k}_{2}$ relates the IKB's volumetric strain to the axial strain measured along the loading direction.

In order to calculate the dislocation densities from Eqs. 5 and 6 , the values of $\beta, \mathrm{k}_{1}$ and $\mathrm{k}_{2}$ need to be estimated. F\&S showed that for the two dimensional case:

$$
2 \beta \approx \frac{2 \alpha(1-v)}{G \gamma_{c}} \frac{\sigma}{M}
$$

For a stress of $450 \mathrm{MPa}, \mathrm{a} \gamma_{\mathrm{c}}$ of 0.012 and $2 \alpha=25 \mu \mathrm{m}$, then $2 \beta \approx 1.8 \mu \mathrm{m}$. 
Eq. 7, is modified from the original equation by replacing the shear stress at the single crystal level by $\sigma / \mathrm{M}$ to account for the polycrystalline case examined here. The values of $\mathrm{k}_{1}$ and $\mathrm{k}_{2}$ range from 1 to 2.[4-6] Herein we assume both to be 1.5. For a random polycrystalline sample, $\mathrm{M}$ is $\approx 3$.[3] For the oriented microstructures examined herein, we assume $\mathrm{M}$ for the $\mathrm{N}$-microstructure to be slightly larger at 3.5. It is worth noting here that the differences in the stress-strain responses between the two samples can be traced to differences in their textures as evidenced by the different stress-strain curves that the EPSC model predicts (compare experimental results and EPSC predicted curves in Fig. 9).

For the microstructures used in this work, if one assumes that the total nonlinear strain for the $\mathrm{N}$-microstructure (0.0017) are due entirely to either DPs, 2D IKBs or prolate spheroid IKBs, the respective dislocation densities, according to Eqs. 4, 5 and 6 , are: $\approx 6.6 \times 10^{10} \mathrm{~m}^{-2}$, $1.2 \times 10^{13} \mathrm{~m}^{-2}$, and $1.4 \times 10^{13} \mathrm{~m}^{-2}$. The uncertainty estimated for these values is $\sim 50 \%$ arising from the large spread in $\alpha$ values. From these calculations it is clear that the calculated $\rho_{\mathrm{DP}}$ $\left(6.6 \times 10^{10} \mathrm{~m}^{-2}\right)$ is almost 3 orders of magnitude less than the density (between $8(2) \times 10^{12}$ and $7(2) \times 10^{13} \mathrm{~m}^{-2}$ ) needed to explain the observed $\{0004\}$ peak widening (Fig. 8). In contradistinction, the dislocation densities calculated for the IKB mechanisms, i.e. assuming $\gamma_{c}$ of $0.012 \mathrm{rad}$, are deep within the range estimated from our experimental results.

Before proceeding much further it is important to point out what the aforementioned equations assume or do not assume. The non-linear strains due to DPs are assumed to be due to their nucleation, presumably from Frank-Read sources, and their ultimate annihilation when the load is removed. Since there is no known mechanism by which this can occur, one must dig deeper. A more realistic analysis would be to start with a given dislocation density, i.e. to assume dislocations do not annihilate but just bow, and then calculate the average distance each dislocation has to move to account for the reversible strain measured.

Sometimes, a small permanent strain is recorded during the first cycle when the MAX phases are loaded in compression.[27] In most cases, however, this permanent strain is quite small. For the sake of argument, we will assume - conservatively - that the permanent strain recorded in the first cycle, is of the same order of magnitude as the nonlinear strain for the Nsample, viz. 0.0017. That in turn would imply that $\approx 7 \times 10^{10} \mathrm{~m}^{-2}$ dislocations in the form of DPs are nucleated during the first cycle. Given that the reversible, non-linear strain after the first cycle is of the same order, then the average distance, $\bar{x}$, these dislocations would have to move reversibly, or bow, is given by:

$$
\bar{x}=\frac{\gamma}{b \rho_{D P}} \approx \frac{\varepsilon_{N L}}{2 b \rho_{D P}}
$$

It follows that for a dislocation density of $6.6 \times 10^{10} \mathrm{~m}^{-2}, \bar{x}$ is of the order of $40 \mu \mathrm{m}$, a value comparable to the size of the grains in our samples (Fig. 2). These calculations - together with all the other evidence presented herein - should put to rest that DPs alone can explain the results such as those obtained herein as some have recently claimed.[18]

Quite recently, Benitez et al. have shown that after cyclically loading fine- and coarsegrained polycrystalline $\mathrm{Ti}_{2} \mathrm{AlC}$ samples in compression, a large concentration of LAKBs were nucleated throughout the microstructure, where none existed before and only after a permanent plastic deformation of less than $0.01 \%$ ! [28] The average angle of these LAKBs was found to be $\approx 5^{\circ}$. If one assumes that on average one $5^{\circ}$ LAKB is nucleated per grain (with $25 \times 60 \times 60$ 
$\mu \mathrm{m}^{3}$ dimensions), during the first cycle - a plausible assumption based on the EBSD results shown - the resulting dislocation density, $\rho_{\mathrm{LAKB}}$, is of the order of $4.8 \times 10^{12} \mathrm{~m}^{-2}$. We further assume the latter are pinned at an average distance of $\mathrm{L}_{\mathrm{N}}$ and that at the maximum stress, each segment bows to a new length given by $2 \pi \mathrm{L}_{N} / 2$, then the change in dislocation density upon application of the maximum stress is simply a factor of $\pi$ times the original $\rho_{\text {LAKB }}$ value. In other words, the change in $\rho_{\mathrm{LAKB}}$, upon loading, is roughly $\pi$ times the original, or preexisting, dislocation density in the form of LAKBs, i.e. $\sim 1.5 \times 10^{13} \mathrm{~m}^{-2}$. The final dislocation density, viz. $7(2) \times 10^{13} \mathrm{~m}^{-2}$, would thus be in the same order of magnitude of the measured value. Needless to say this agreement is fortuitous and depends on many assumptions, some better than others. For example, there is no reason to believe that on average one $5^{\circ} \mathrm{LAKB}$ is nucleated per grain. The point of this exercise, however, is to make the point that the bowing of LAKB dislocations cannot be excluded as a viable mechanism at this time. At this juncture it is important to note that Roberts and coworkers [29, 30] observed similar fully reversible loops in $\mathrm{Zn}$ and $\mathrm{Mg}$ and attributed them, without much evidence, to the bowing out of dislocations.

The fundamental difference between DPs and KBs is in how their dislocations are arranged. Slip by DPs occurs by the successive generation of many dislocations all in the same slip plane; kinking occurs by the generation of kink boundaries in which dislocations are nucleated on many parallel slip planes separated by a distance, D (Fig. 1(d)).[25] In other words, since in DPs there are many dislocations of the same sign on the same slip plane, their stress fields add up and result in relatively large strains. However, in KBs same sign dislocations lie above one another, such that their stress fields partially cancel resulting in less strain. As a result, it is possible to have much higher dislocation densities in the form of KBs. We emphasize once more that it is not possible to reconcile the results shown in this work with the presence of dislocations arranged only in DPs; KBs of some sort have to be invoked.

\section{EPSC Modeling}

The inputs to the EPSC model are the: i) elastic constants, $\mathrm{c}_{\mathrm{ij}}$ 's; ii) critical resolved shear stresses, CRSS, of mobile dislocations - which for the MAX phases are exclusively basal, and, iii) hardening rates and their stress dependencies.

To date the only elastic constants for $\mathrm{Ti}_{2} \mathrm{AlC}$ available are those determined from density functional theory, DFT. Table 1 lists the results of six such studies for $\mathrm{Ti}_{2} \mathrm{AlC}$. The values used herein are listed in the bottom of the table and represent the average of the six studies. We note in passing that the response of $\mathrm{Ti}_{2} \mathrm{SC}$, a MAX phase that deforms in a liner elastic fashion, was perfectly modeled by EPSC starting with the results of ab initio studies.[31]

Table 2 lists the parameters used by the EPSC approach to model both the bulk stressstrain curves and the response of the various reflections. The inputs include the critical resolved shear stress, CRSS assumed to be $35 \mathrm{MPa}$ and various hardening rates. For the $\mathrm{N}$ sample a constant hardening rate, HR, of $5.5 \mathrm{MPa}$ was assumed. To fit the P-sample, however, assuming a CRSS of $35 \mathrm{MPa}$, the HR had to decrease from $23 \mathrm{MPa}$ to $9.5 \mathrm{MPa}$ as the resolved shear stress increased from $35 \mathrm{MPa}$ to $50 \mathrm{MPa}$.

Table 1: $\mathrm{Ti}_{2} \mathrm{AlC}$ elastic constants from ab initio calculations (see Refs. [32-37]). 


\begin{tabular}{|c|c|c|c|c|c|c|c|}
\hline & $\mathrm{c}_{11}$ & $\mathrm{c}_{12}$ & $\mathrm{c}_{13}$ & $c_{33}$ & $\mathrm{c}_{44}$ & $\mathrm{c}_{66}$ & \multirow{7}{*}{$\begin{array}{c}\text { Reference } \\
\text { (Rosen et al., 2010) } \\
\text { (Du et al., 2009) } \\
\text { (Wang el al., 2004) } \\
\text { (Sun et al., 2004) } \\
\text { (Bouhemadou et al., 2007) } \\
\text { (Cover et al., 2009) }\end{array}$} \\
\hline & 305 & 60 & 60 & 275 & 110 & 122.5 & \\
\hline & 302 & 59 & 55 & 278 & 113 & 121.5 & \\
\hline & 308 & 55 & 60 & 270 & 111 & 126.5 & \\
\hline & 321 & 76 & 100 & 318 & 144 & 122.5 & \\
\hline & 307 & 58 & 63 & 284 & 118 & 124.5 & \\
\hline & 302 & 62 & 61 & 269 & 109 & 120 & \\
\hline $\begin{array}{c}\text { Average } \\
\text { Values Used }\end{array}$ & 307.5 & 61.7 & 66.5 & 282.3 & 117.5 & 122.9 & \\
\hline Standard Deviation & 7.1 & 7.4 & 16.6 & 18.3 & 13.4 & 2.3 & \\
\hline
\end{tabular}

Table 2: Parameters used in the EPSC calculations assuming dislocation pileups, with a critical resolved shear stress, CRSS of $35 \mathrm{MPa}$, are responsible for the deformation observed. To fit the bulk stress-strain curves of the N-sample a constant hardening rate, HR, of 5.5 MPa was assumed. To fit the P-sample, assuming a CRSS of $35 \mathrm{MPa}$, the HR had to decrease from 23 $\mathrm{MPa}$ to $9.5 \mathrm{MPa}$ as the resolved shear stress increased from $35 \mathrm{MPa}$ to $50 \mathrm{MPa}$.

\begin{tabular}{|c|c|c|c|c|c|}
\hline Microstructure & Initial \# of grains & CRSS (MPa) & Gradual (MPa) & HR1 (MPa) & HR2 (MPa) \\
\hline Normal & 15,552 & 35 & - & 5.5 & 5.5 \\
\hline Parallel & 15,552 & 35 & 15 & 23 & 9.5 \\
\hline
\end{tabular}

At this stage it is instructive to break down the discussion related to the EPSC fits into three subsections viz.: i) bulk stress-strain results, and single peak fits of, ii) all but $\{0001\}$ and $\{10 \overline{1} 0\}$ reflections, and, iii) $\{0001\}$ and $\{10 \overline{1} 0\}$ reflections.

i) Bulk stress strain curves. As noted above, to fit the bulk stress-strain curves of the Nsample a constant hardening rate, HR, of 5.5 MPa was assumed in the EPSC model. When the same parameters were used to fit the P-sample, the fits were quite poor (see open crosses in Fig. 9). To fit the P-sample, assuming the same CRSS, the HR was assumed to change from 23 $\mathrm{MPa}$ to $9.5 \mathrm{MPa}$ as the resolved shear stress increased from 35-50 MPa (see Table 2). The resulting fit is shown in Figs. 1(b) and 9. Such large changes in HRs are physically untenable in two samples that are identical in all respects, but the orientation of the grains relative to the loading direction. Said otherwise, the fact that the same parameters cannot fit the bulk stressstrain curves of both microstructures can be taken as indirect evidence that DPs alone cannot explain our results. This is especially true since the EPSC model uses the pole figures generated for the two microstructures into account.

ii) Response of the non- $\{0001\}$ and non $-\{10 \overline{1} 0\}$ reflections

The response of all but the $\{0001\}$ and $\{10 \overline{1} 0\}$ reflections on loading is reasonably well captured by the EPSC model assuming DPs are activated. This is best exemplified by the $\{10 \overline{1} 7\}$, $\{10 \overline{1} 5\}$ and $\{10 \overline{1} 1\}$ reflections (Fig. 10(a)), the former two are clearly plastically deforming, presumably by DPs. The $\{10 \overline{1} 7\}$, and $\{10 \overline{1} 5\}$ reflections are from soft grains whose basal planes are inclined $\sim 33^{\circ}$ and $\sim 42^{\circ}$ to the loading direction, respectively. The $\{10 \overline{1} 1\}$ lattice plane reflections (depicted in Fig. 10(a) by blue triangles), however, are from a harder grains 
whose basal planes are inclined $\sim 13^{\circ}$ to the loading direction. These results are also consistent with the activation of basal slip, since the stress no longer carried by the soft grains - because they deformed - is redistributed unto the harder $\{10 \overline{1} 1\}$ reflections, that originate in grains with lower Schmid factors.

The response of the P-sample in the transverse direction (Figs. 10(b) and (c)) is also in total agreement with this scheme. The response shown in Fig. 10(c) is noteworthy and would be very difficult to interpret without the EPSC model. But according to the model, this is the response such reflections would behave if basal slip were activated. The latter statement, however, is only true upon loading.

The agreement upon unloading is, not surprisingly, bad, and is attributable to the fact that the EPSC model does not model IKBs or dislocations in LAKBs that bow. What the EPSC model predicts instead is the presence of large residual elastic strains upon full unloading that are not observed experimentally. Not surprisingly, the ND results show fully reversible behavior that mimics the bulk stress-strain curves instead.

To summarize: All reflections - except $\{0001\}$ and $\{10 \overline{1} 0\}$ - on loading only, can be qualitatively and quantitatively predicted by the EPSC model in which only basal slip is activated (Figs. 5-7 and 10). Since these reflections emerge from grains whose basal planes form different angles with the loading directions, they represent different degrees of "hardness".

ii) Response of $\{0001\}$ and $\{10 \overline{1} 0\}$ reflections

These reflections were already discussed briefly above. Given their importance, however, they are discussed here again in light of the EPSC model results. In the remainder this paper we make the case that the response of the $\{0001\}$ reflections is inconsistent with the existence of DPs in these grains.

Referring to Figure 7, the EPSC model predicts correctly that the deviation from linear elastic of these, the hardest grains, should be the most extreme, with concomitant residual strains of the order of - $600 \mu$ strain. Experimentally that is far from the case for both the longitudinal $\{0004\}$ reflections in the $\mathrm{N}$-sample and the corresponding reflections in the transverse direction (Fig. 7). The inescapable conclusion is that a micromechanism, that is not DP-based, is activated in these grains that reduces the stresses and strains they experience.

Before presenting further evidence for the existence for kink boundaries, it is important to eliminate some common sources of mechanical hysteresis that could explain our results such as the Bauschinger effect, microcracking, reversible twinning, etc. Quite recently, stress-strain hysteresis loops of austenitic stainless steel samples were observed in SMARTS and attributed to the Bauschinger effect.[12] Along the same lines, twinning can be reversed in some situations.[38] In both cases, however, full reversibility is achieved only by reversing the loading direction. Herein the reversibility is full and spontaneous, a most crucial distinction indeed.

Microcracking can also result in hysteretic bulk stress-strain curves, especially in ceramics.[39] For example, in a recent SMARTS study on porous alumina - with porosities as high as 47 vol.\% - small loops were only observed at the highest porosities.[40] Given that alumina is significantly more brittle than $\mathrm{Ti}_{2} \mathrm{AlC}$, it is unlikely that the hysteresis loops observed herein are due to porosity and/or microcracking, especially since the samples used 
herein were fully dense. In another SMARTS study on rock samples[41] bulk hysteresis loops were observed and related to microcracks. However, opposite of what is observed here, the stress-strain curves traced counter-clockwise loops. As important, at the microscopic, i.e. ND level, the response was fully linear elastic in all directions,[41] again, in stark contrast to the results shown in Figs. 5, 6 and 10.

At this point it is reasonable to conclude that the behavior observed herein cannot be primarily a result of microcracking, twins and/or DPs alone. Another mechanism is needed. The latter must: a) explain the fully reversible, but energy dissipative stress-strain loops, both the macroscopic (Fig. 1b), and microscopic (Figs. 5-7 and 10); b) account for the unusually large energy stored at maximum stress that in turn creates the driving force necessary to spontaneously reverse the non-linear deformation, and; c) generate a profusion of KBs upon deformation, especially at higher temperatures or stresses. Henceforth we argue that IKB formation normal to the basal planes and/or the bowing of LAKB dislocations is the missing piece of the puzzle.

A good example is the scanning electron microscope micrograph shown in Fig. 11, of a fractured surface of a $\mathrm{Ti}_{2} \mathrm{AlC}$ sample that was crept to failure at $1100{ }^{\circ} \mathrm{C}$. [42] The high density of KBs support the notion that a multiplicity of LAKBs form in the MAX phases upon deformation. The grip areas' fractured surfaces (inset in Fig. 11), were devoid of such kinks. We note in passing that it is difficult to imagine how DPs could produce the KBs shown in Fig. 11.

In conclusion, careful analysis of in situ ND results of polycrystalline textured $\mathrm{Ti}_{2} \mathrm{AlC}$ samples, loaded in compression to $450 \mathrm{MPa}$, is consistent with kinking. All lattice strains are reversible, showing pronounced hysteresis loops of select reflections. It is crucial to note here that in the $\mathrm{N}$-sample, at least some of the hard grains must deform against their strain energy considerations when loaded. This comes about because when the latter kink, they do so with a strain component opposite to the loading direction. Said otherwise, the grains expand along the compression axis. Such contrarian deformations, at the individual grain level, have previously been observed in the MAX phases,[43] and in polycrystalline $\mathrm{Mg}$ [44], and simply cannot be explained by DPs. We note in passing that in a recent quite careful ND study on polycrystalline $\mathrm{Mg}$ some aspects of the deformation were speculated to be due to the formation of KBs.[38]

Hopefully, as a result of this work, the important role LAKBs and IKBs play in the early deformation of solids will be better appreciated. This is important because the ramifications and implications of their existence is far-reaching and profound. Of all fields, probably the one that would be most directly impacted is geology. Over the past decade we have shown that mica - and by extension all other layered silicates and most probably ice - is a KNE solid.[45, 46] It follows that not accounting for LAKBs and/or IKBs could, in principle, underestimate the energy stored in geologic formations by a factor approaching two. As importantly, we have recently shown - unpublished results - that, when nucleated, IKBs can substantially increase the damping of ultrasound. Accounting for the latter could in principle, render ultrasound measurements of the earth's crust and their interpretation more accurate.

In the field of metallurgy, we have shown that KBs can explain microyielding and damping in technologically important hexagonal metals, such $\mathrm{Ti}, \mathrm{Mg}, \mathrm{Co}, \mathrm{Zn}$ and $\mathrm{Zr}$.[5] Further, it is reasonable to assume that IKB and/or LAKBs, because they are nucleated at quite low strains, most probably play an important role in the overall development of dislocation networks observed at higher plastic strains. Said otherwise, it is only by understanding the 
dislocation networks at low strains that the ones at higher strains can be fully explained. This is especially true if it can be shown that, for example, IKBs can nucleate twins. We have also shown that graphite, a technologically important material, is a KNE solid [45]. Prior to our work, damping and microyielding in graphite were attributed to DPs. However, as discussed herein, DPs cannot account for our observations. Whether LAKB and/or IKB nucleation is favored or hindered in high neutron flux environments is an important question that needs to be looked into.

Lastly, since the only requirement for a hexagonal solid to be KNE is a high c/a ratio, the latter are ubiquitous in nature. It follows that LAKBs and/or IKB's play an important, but hitherto unappreciated, role in the deformation of KNE solids in general and in their early deformation in particular.

\section{Acknowledgements:}

This work was supported by the Army Research Office (No. W911NF-07-1-0628). This work has benefited from the use of the Lujan Neutron Scattering Center at LANSCE, funded by the Department of Energy's Office of Basic Energy Sciences. Los Alamos National Laboratory is operated by Los Alamos National Security LLC under DOE Contract DE-AC5206NA25396. Use of the equipment of the Centralized Research Facility (Drexel University) is acknowledged. V.P. acknowledges financial support by the Alexander von Humboldt foundation.

\section{Author contributions}

E. N. Caspi took the lead in analyzing the ND diffraction results; M. Shamma generated all the EPSC predictions; B. Clausen, D. Brown and S. Vogel are the instrument scientists that collected the ND results on HIPPO and SMARTS; S. Amini fabricated the samples, S. Vogel analyzed the HIPPO results, V. Presser and O. Yeheskel were involved in analyzing the ND results at various stages of the project. B. Anasori helped develop the model, especially the 2D one; O. Yeheskel and E. N. Caspi were also involved in numerous discussions of the results. M. W. Barsoum conceived and directed the project. 


\section{Appendix A}

Equation 3 was derived assuming a dislocation line energy of $\mathrm{Gb}^{2} / 2$. This line energy is for isolated dislocations that have long-range elastic field that do not interact. To estimate the reduction in energy due to the fact that the dislocations are in a wall, we start with our previous work [47] where we used DFT to calculate the energies of the a kink boundary, where a dislocation is present every c-lattice parameter, c. Taking $1 \mathrm{~m}^{2}$ as our basis, we estimate the total energy of non-interacting dislocation, whose density is $1 / \mathrm{c}$, to be

$$
\mathrm{E}_{\text {non-interac }}=\mathrm{Gb}^{2} / 2 \mathrm{c}
$$

For $\mathrm{Mg}$ and $\mathrm{Be}, \mathrm{E}_{\text {non-interac }}$ is thus $2.65 \mathrm{~J} / \mathrm{m}^{2}$ and $18.24 \mathrm{~J} / \mathrm{m}^{2}$, respectively. The respective surface energies calculated from DFT, however, are 0.122 and $1.82 \mathrm{~J} / \mathrm{m}^{2}$. The ratio between the two is the order of 20 for $\mathrm{Mg}$, and 10 for $\mathrm{Be}$.

In our case, the distance between dislocations is not $\mathrm{c}$, but larger and depends on the kink angle, $\gamma_{\mathrm{c}}$. For a $\gamma_{\mathrm{c}}$ of $0.012, \mathrm{D}=18 \mathrm{c}$ and it is reasonable to assume that the dislocations in the KBs are weakly interacting and thus $\phi$ can be assumed to be $\approx 1$. However, if the kink boundary angle is $5^{\circ}$, on average, then the distance between dislocations is of the order of $2.5 \mathrm{c}$. Interpolating between a value of 1 for infinitely separated dislocations and 20 for ones that are c-apart (like in the case of $\mathrm{Mg}$ ), we obtain a value of $\approx 8.6$ which we round to 9 .

\section{$\underline{\text { Appendix B }}$}

\section{Nonlinear Strains and Dislocation Densities}

\section{Dislocation Pileups}

The energy stored - per unit length measured along their lines - in one pileup with $\mathrm{n}$ dislocations is given by: ${ }^{[48]}$

$$
U_{L}=\frac{G b^{2}}{4 \pi(1-v)} n^{2} \ln [4 \sqrt{e}]
$$

where $\mathrm{G}, \mathrm{b}$ and $\mathrm{v}$ are the shear modulus, Burgers vector and Poisson's ratio. Further assuming, ${ }^{[48]}$

$$
n=\frac{\pi \lambda(1-v)}{G b} \tau
$$

where $\tau$ is the applied shear stress and $\lambda$ the grain diameter. Converting to an energy per unit volume then:

$$
U_{D P}=\frac{G b^{2}}{4 \pi(1-v)} 2 \pi \lambda_{a v} N_{D P} \frac{\pi^{2} \lambda^{2}(1-v)^{2}}{G^{2} b^{2}} \tau^{2} \ln (4 \sqrt{e})
$$




$$
U_{D P}=\frac{\pi^{2} \lambda^{2} \lambda_{a v}(1-v)}{2 G} N_{D P} \tau^{2} \ln (4 \sqrt{e})
$$

where $\mathrm{N}_{\mathrm{DP}}$ is the number of DP per unit volume and $\lambda_{\mathrm{av}}$ is the average length of a dislocation in a pileup. But since $U_{D P}=1 / 2 \gamma_{D P} \tau$, where $\tau$ is applied shear stress and $\gamma_{\mathrm{DP}}$ is the shear strain then:

$$
\gamma_{D P}=\frac{\pi^{2} \lambda^{2} \lambda_{a v}(1-v)}{G} N_{D P} \ln (4 \sqrt{e}) \tau
$$

Assuming $\lambda_{\mathrm{av}}=\lambda / 3$, then:

$$
\gamma_{D P}=\frac{1.89 \pi^{2} \lambda^{3}(1-v)}{3 G} N_{D P} \tau
$$

Further assuming, $\tau=\sigma / \mathrm{M}$ and $\gamma_{\mathrm{DP}}=2 \varepsilon_{\mathrm{DP}}(1+v) / \mathrm{M}$, then $\mathrm{B} 5$ can be recast to read:

$$
\varepsilon_{D P}=\frac{1.89 \pi^{2} \lambda^{3}(1-v)}{6 G(1+v)} N_{D P} \sigma
$$

The corresponding dislocation density associated with this strain is:

$$
\rho_{D P}=2 \pi \frac{\lambda}{3} n N_{D P}
$$

Combining Eqs. B2, B6 and B7, it can shown that:

$$
\rho_{D P} \approx \frac{2.1(1+v) \varepsilon_{D P}}{M b \lambda}
$$

\section{Prolate Spheroids}

This IKB consists of multiple parallel dislocation loops (inset in Fig. 1(a)). As a first approximation each loop can be assumed to be made up of two edge and two screw dislocation segments with lengths, $2 \beta_{\mathrm{x}}$ and $2 \beta_{\mathrm{y}}$, respectively. The latter are assumed to be related to the applied stress, $\sigma$ and $2 \alpha$ by [25]:

$$
2 \beta_{\mathrm{x}} \approx \frac{2 \alpha(1-v)}{\mathrm{G} \gamma_{\mathrm{c}}} \frac{\sigma}{\mathrm{M}} \quad \text { and } \quad 2 \beta_{\mathrm{y}} \approx \frac{2 \alpha}{\mathrm{G} \gamma_{\mathrm{c}}} \frac{\sigma}{\mathrm{M}}
$$

Note that these expressions were modified from the original F\&S paper to account for the polycrystalline nature of the materials tested herein by the introduction of $\mathrm{M}$. In the remaining of this discussion the simplifying assumption that $\beta_{x}=\beta_{y}=\beta$ is made.

The formation of an IKB can be divided into two stages: nucleation and growth.[2] Since the former is not well understood, our model only considers IKB growth from $2 \beta_{\mathrm{xc}}$ and $2 \beta_{\mathrm{yc}}$ to $2 \beta_{\mathrm{x}}$ and $2 \beta_{\mathrm{y}}$, respectively. The dislocation segment lengths of an IKB nucleus, $\beta_{\mathrm{xc}}$ and $2 \beta_{y c}$, are presumed to either pre-exist, or are nucleated during pre-straining. We note in passing that at this time, and based on a number of studies on KNE solids,[2] it appears that some prestraining is needed to observe KNE behaviour.

Frank and Stroh[25] derived the following equation for the stress at which an IKB in a 
2D crystal would become unstable and run to the end of the crystal:

$$
\tau_{c} \approx \frac{\sigma_{c}}{M}=\sqrt{\frac{G^{2} b \gamma_{c}}{(1-v)^{2} \pi^{2} 2 \alpha} \ln \frac{b}{w \gamma_{c}}}
$$

It follows that for $\sigma>\sigma_{\mathrm{c}}$, the IKB nuclei grow and the IKB-induced axial strain resulting from their growth is assumed to be given by:[2]

$$
\varepsilon_{I K B}=\frac{\Delta V \gamma_{c}}{k_{1}} N_{I K B}=\frac{\gamma_{c} 4 \pi \alpha \beta^{2}}{3 k_{1}} N_{I K B}
$$

where NIKB is the number of IKBs per unit volume; $\Delta \mathrm{V}$ is change in the volume kinked as the IKBs grow from their critical size at $\sigma_{\mathrm{c}}$ to their size at $\sigma$. It follows that the volume fraction of the material that is kinked, $\mathrm{v}_{\mathrm{f}}$, is given by the product $\mathrm{V} \cdot \mathrm{N}_{\mathrm{IKB}}$. The factor $\mathrm{k}_{1}$ relates the volumetric strain due to the IKBs to the axial strain along the loading direction.

If one assumes that the average radii of the dislocation loops along the height of a prolate spheroid IKB, $\beta_{\mathrm{av}}$, is $\beta / 2$ the dislocation density is given by:

$$
\rho_{I K B} \approx 2 \pi \frac{\beta}{2} \frac{2 \alpha}{D} N_{I K B}
$$

Combining this equation with (B11), and Eq. 2, yields Eq. 5 in the text.

\section{Two-dimensional IKBs}

This is the original IKB - shown schematically in Fig. 1(d) - postulated by F\&S.

In this case,

$$
\varepsilon_{2 D}=\frac{\Delta V \gamma_{c}}{k_{2}} N_{2 D}=\frac{\gamma_{c} \pi \alpha \beta \lambda}{k_{2}} N_{2 D}
$$

where $\mathrm{N}_{2 \mathrm{D}}$ is the number of two dimensional IKBs per unit volume. The corresponding dislocation density is given by:

$$
\rho_{2 D} \approx \frac{2 \alpha}{D} 2 \lambda N_{2 D}
$$

Combining the two equations and Eq. 2, results in Eq. 6. 
Figure Captions:

Figure 1: a) Schematic of fully reversible stress-strain curves showing the various parameters used in the IKB model. Inset shows schematic of prolate spheroid IKB formed on parallel basal planes with a spacing of D between them; b) Bulk stress-strain curves for sample whose basal planes are normal "N" (blue squares), or perpendicular "P" (red circles) to the compressive loading direction. Both stress and strain axes are reversed to render the plots similar to conventional tensile stress-strain curves. Schematics of, (i) P- and, (ii) N-microstructure. In these schematics the thin lines represent basal planes. The black and red rectangles represent the majority and minority hard grains, respectively. The blue rectangles represent the soft grains. c) Schematic of sample configuration relative to the neutron beam, detectors (Bank 1 - Transverse; Bank 2 - Longitudinal), and loading direction in SMARTS. d) Modified subcritical 2dimensional IKB proposed by Frank and Stroh.

Figure 2: Cross-sectional polished and etched optical microscope, OM, image of microstructure. White areas are $\mathrm{TiC}$ and dark areas are pores resulting from the pull-out of $\mathrm{TiC}$ grains.

Figure 3: Rietveld analysis of ND results of the N-sample as measured on the SMARTS diffractometer, under a compressive load corresponding to a stress of $5 \mathrm{MPa}$. These results were collected using bank 2, configured to measure the longitudinal stress direction (see Fig. 1(c)). Open circles, solid line, and dashed line in the bottom, represent the measured data, refined model, and the difference between the two, respectively. The three rows of vertical tags represent the calculated Bragg reflections' positions of the major $\mathrm{Ti}_{2} \mathrm{AlC}$ ( ${ }^{\text {st }}$ row), and the minor $\mathrm{Ti}_{3} \mathrm{AlC}_{2}\left(2^{\text {nd }}\right)$, and $\mathrm{Ti}_{5} \mathrm{Al}_{2} \mathrm{C}_{3}\left(3^{\text {rd }}\right)$ phases. The strong preferred orientation of the $\{000 l\}$ reflections is marked for the major $\mathrm{Ti}_{2} \mathrm{AlC}$ phase. In this fit $\chi^{2}=2.489$.

Figure 4: Pole figures for, a) $\{0002\}$ and, b) $\{10 \overline{1} 5\}$ planes of N-sample; c) $\{0002\}$ and, d) $\{10 \overline{1} 5\}$ planes of $\mathrm{P}$-sample.

Figure 5: Stress-strain response obtained from single peak fits of $\{10 \overline{1} 5\},\{10 \overline{1} 7\},\{10 \overline{1} 2\}$ and $\{10 \overline{1} 1\}$ reflections along, a) transverse and, b) longitudinal directions for the N-sample and, c) transverse and d) longitudinal directions for the P-orientation. Results obtained on loading are represented by solid symbols; those on unloading by open ones. The EPSC model results, assuming linear elastic behavior (green dashed lines). Both axes are reversed to render the plots similar to conventional tensile stress-strain curves.

Figure 6: Stress-strain response obtained from single peak fits of $\{0001\},\{10 \overline{1} 0\}$ and $\{10 \overline{1} 5\}$ reflections along, a) transverse and, b) longitudinal directions for the N-sample and, c) transverse and d) longitudinal directions for the P-orientation. Results obtained on loading are represented by solid symbols; those on unloading by open ones. The EPSC model results, assuming linear elastic behavior (green dashed lines), and basal slip (dotted red lines). The various curves are shifted for clarity. Both axes are reversed to render the plots similar to conventional tensile stress-strain curves.

Figure 7: Stress-strain response of $\{0004\}$ reflections along transverse (red data points) direction in P-sample (blue data points) in the longitudinal direction of the N-sample. The 
predicted response has DPs been activated is given by the dotted black line. Both axes are reversed to render the plots similar to conventional tensile stress-strain curves.

Figure 8: Full width at half maximum (FWHM) of the N-sample $\{0004\}$ ND reflections on the longitudinal bank as a function of stress. Note hysteresis. Inset shows FWHM for the $\{10 \overline{1} 0\}$ reflections in the P-sample.

Figure 9: Fit of P-sample bulk stress-strain curves (purple crosses) assuming the same parameters used for N-sample, viz. CRSS $=35 \mathrm{MPa}$ and $\mathrm{HR}=5.5 \mathrm{MPa}$. The fit is clearly quite poor. To obtain the better fit shown as a solid black line, the hardening rates had to be increased to the values listed in Table 2. The experimental results for the $\mathrm{N}$-sample are shown as squares; for the P-sample as open circles. Both axes are reversed to render the plots similar to conventional tensile stress-strain curves.

Figure 10: Stress-strain response obtained from single peak fits of the ND results for, a) $\{10 \overline{1} 5\}$, $\{10 \overline{1} 7\}$ and $\{10 \overline{1} 1\}$ reflections along the longitudinal and, b) $\{10 \overline{1} 5\}$ reflections along the transverse direction both for the N-oriented sample. c) $\{10 \overline{1} 1\}$ and $\{10 \overline{1} 5\}$ reflections along transverse direction of P-oriented sample. d) $\{10 \overline{1} 1\}$ and $\{10 \overline{1} 5\}$ reflections along longitudinal direction of P-oriented sample. The predicted response assuming DPs (linear eastic) been activated is given by the dotted (green dashed) lines. Both axes are reversed to render the plots similar to conventional tensile stress-strain curves.

Figure 11: SEM micrograph of fractured surface of a $\mathrm{Ti}_{2} \mathrm{AlC}$ sample held at $1100^{\circ} \mathrm{C}$ and 30 MPa for $3 \mathrm{~h}$ that failed at a tensile strain of $16 \%$. This micrograph emphasizes the profusion of kink boundaries formed as a result of the deformation. Inset shows a fractured surface of the grip area where no kink boundaries are present [42]. 


\section{References:}

[1] S. Chakraborty, T. El-Raghy, M.W. Barsoum. Oxidation of $\mathrm{Hf}_{2} \mathrm{SnC}$ and $\mathrm{Nb}_{2} \mathrm{SnC}$ in Air in the 400-600 ${ }^{\circ} \mathrm{C}$ Temperature Range, Oxid. Metals 59 (2003) 83-96

[2] M.W. Barsoum, S. Basu. Kinking Nonlinear Elastic Solids. in: K. H. J. Buschow RWC, M. C. Flemings, B. Ilschner, E. J. Kramer, S. Mahajan and P. Veyssiere, (Ed.). Encyclopedia of Materials Science and Technology. Elsevier, Oxford, 2010. pp. 1-23.

[3] M.W. Barsoum, T. Zhen, A. Zhou, S. Basu, S.R. Kalidindi. Microscale modeling of kinking nonlinear elastic solids, Physical Review B 71 (2005).

[4] A.G. Zhou, M.W. Barsoum, S. Basu, S.R. Kalidindi, T. El-Raghy. Incipient and regular kink bands in fully dense and 10 vol.\% porous Ti2AlC, Acta Materialia 54 (2006) 1631-1639.

[5] A.G. Zhou, B. Basu, M.W. Barsoum. Kinking Nonlinear Elasticity, Damping and Microyielding of Hexagonal Close-Packed Metals, Acta Mat. 56 (2008) 60-67.

[6] A. Zhou, M.W. Barsoum. Kinking Nonlinear Elasticity and the Deformation of Mg, Metall. Mater. Trans. 40A (2009) 1741-1756.

[7] A.G. Zhou, M.W. Barsoum. Kinking nonlinear elastic deformation of Ti3AlC2, Ti2AlC, $\mathrm{Ti} 3 \mathrm{Al}(\mathrm{C} 0.5, \mathrm{~N} 0.5) 2$ and Ti2Al(C0.5,N0.5), Journal of Alloys and Compounds 498 (2010) 62-70.

[8] P.A. Turner, C.N. Tomé. A study of residual stresses in Zircaloy-2 with rod texture, Acta Metallurgica et Materialia 42 (1994) 4143-4153.

[9] B. Clausen, T. Lorentzen, T. Leffers. Self-consistent modelling of the plastic deformation of f.c.c. polycrystals and its implications for diffraction measurements of internal stresses, Acta Materialia 46 (1998) 3087-3098.

[10] B. Clausen, T. Lorentzen, M.A.M. Bourke, M.R. Daymond. Lattice strain evolution during uniaxial tensile loading of stainless steel, Materials Science and Engineering A 259 (1999) 17-24.

[11] B. Clausen, C.N. Tomé, D.W. Brown, S.R. Agnew. Reorientation and stress relaxation due to twinning: Modeling and experimental characterization for Mg, Acta Materialia 56 (2008) 2456-2468.

[12] J.A. Wollmershauser, B. Clausen, S.R. Agnew. A slip system-based kinematic hardening model application to in situ neutron diffraction of cyclic deformation of austenitic stainless steel, International Journal of Fatigue 36 (2012) 181-193.

[13] M.W. Barsoum. The MN+1AXN phases: A new class of solids : Thermodynamically stable nanolaminates, Progress in Solid State Chemistry 28 (2000) 201-281.

[14] T. Zhen, M.W. Barsoum, S.R. Kalidindi. Effects of Temperature, Strain Rate and Grain Size on the Compressive Properties of $\mathrm{Ti}_{3} \mathrm{SiC}_{2}$, Acta Mat. 53 (2005) 4163-4171.

[15] Y. Gogotsi, A. Nikitin, H. Ye, W. Zhou, J.E. Fischer, B. Yi, H.C. Foley, M.W. Barsoum. Nanoporous Carbon-derived Carbon with Tunable Pore Size, Nature Materials 2 (2003) 591-594.

[16] T. Zhen, M.W. Barsoum, S.R. Kalidindi. Effects of temperature, strain rate and grain size on the compressive properties of Ti3SiC2, Acta Mater 53 (2005) 4163-4171.

[17] A. Guitton, A. Joulain, L. Thilly, C. Tromas. Dislocation analysis of Ti2AlN deformed at room temperature under confining pressure, Phil. Mag. Letters 92 (2012) 4536-4546.

[18] N.G. Jones, C. Humphrey, L.D. Connor, O. Wilhelmsson, L. Hultman, H.J. Stone, F. Giuliani, W.J. Clegg. On the relevance of kinking to reversible hysteresis in MAX phases, Acta Mater 69 (2014) $149-161$.

[19] H.R. Wenk, L. Lutterotti, S. Vogel. Texture analysis with the new HIPPO TOF diffractometer, Nuclear Instruments \& Methods in Physics Research Section a-Accelerators Spectrometers Detectors and Associated Equipment 515 (2003) 575-588.

[20] H.R. Wenk, L. Lutterotti, S.C. Vogel. Rietveld texture analysis from TOF neutron diffraction data, Powder Diffr. 25 (2010) 283-296.

[21] M.A.M. Bourke, D.C. Dunand, E. Ustundag. SMARTS - a spectrometer for strain measurement in engineering materials, Applied Physics A-Materials Science \& Processing 74 (2002) S1707-S1709.

[22] R.B.L. Von Dreele, A. C. General Structure Analysis System (GSAS), Los Alamos National Laboratory Report LAUR (2004) 86-748. 
[24] B. Clausen. SMARTSware, Los Alamos National Laboratory Report LAUR (2004) 04-6581.

[25] F.C. Frank, A.N. Stroh. On the Theory of Kinking, Proceeding of the Physical Society B 65 (1952) 811-821.

[26] F. Christien, M.T.F. Telling, K.S. Knight. Neutron diffraction in situ monitoring of the dislocation density during martensitic transformation in a stainless steel, Scripta Mater. 68 (2013) 506509.

[27] A.G. Zhou, M.W. Barsoum. Kinking nonlinear elastic deformation of Ti3AlC2, Ti2AlC, Ti3 $\mathrm{Al}(\mathrm{C} 0.5, \mathrm{~N} 0.5) 2$ and Ti2Al(C0.5,N0.5), J. Alloys Compds. 498 (2010) 62-67.

[28] R. Benitez, W. Hao, H. Gao, M. O'Neil, G. Proust , M. Radovic. Room Temperature StressStrain Hysteresis in Ti2AlC Revisited, (Sub for Pub.).

[29] J.M. Roberts, N. Brown. Microstrain in Zinc Single Crystals, Trans. AIME 218 (1960) 454-463.

[30] J.M. Roberts, D.E. Hartman. The temperature dependence of the microyield points in prestrained magnesium single crystals, Tran. AIME 230 (1964) 1125-1133.

[31] M. Shamma, V. Presser, B. Clausen, D. Brown, O. Yeheskel, M.W. Barsoum. On the Response of Ti2SC to Stress Studied by in situ Neutron Diffraction and the Elasto-Plastic Self-Consistent Approach., Scripta Mater. 65, (2011) 573-576

[32] Z. Sun, S. Li, R. Ahuja, J.M. Schneider. Calculated elastic properties of M2AlC (M = Ti, V, Cr, $\mathrm{Nb}$ and Ta), Solid State Communications 129 (2004) 589-592.

[33] J. Wang, Y. Zhou. Dependence of Elastic Stiffness on Electronic Band Structure of Nanolaminate M2AlC (M=Ti,V,Nb and Cr) Ceramics, Phys. Rev. B 69 (2004) 214111.

[34] M.F. Cover, O. Warschkow, M.M. Bilek, D.R. McKenzie. A comprehensive survey of M2AX phase elastic properties, J. Phys.: Condens. Matter 21 (2009).

[35] Y.L. Du, Z.-M. Sun, H. Hashimoto, M.W. Barsoum. Theoretical investigations on the elastic and thermodynamic properties of Ti2AlC0.5N0.5 solid solution, Physics Letters A 374 (2009) 78-82.

[36] A. Bouhemadou, R. Khenata, M. Chegaar. Structural and elastic properties of Zr2AlX and Ti2AlX (X = C and N) under pressure effect, Eur. Phys. J. B 56 (2007) 209-215.

[37] J. Rosen, M. Dahlqvist, S.I. Simak, D.R. McKenzie, M.M.M. Bilek. Oxygen Incorporation in Ti2AlC: Tuning of Anisotropic Conductivity Appl. Phys. Lett. 97 (2010) 073103

[38] O. Muransky, D.G. Carr, P. Sittner, E.C. Oliver. In situ neutron diffraction investigation of deformation twinning and pseudoelastic-like behaviour of extruded AZ31 magnesium alloy, International Journal of Plasticity 25 (2009) 1107-1127.

[39] B.R. Lawn, D.B. Marshall. Nonlinear stress-strain curves for solids containing closed cracks with friction, Journal of the Mechanics and Physics of Solids 46 (1998) 85-113.

[40] G. Bruno, A. Efremov, A. Levandovskyi, B. Clausen. Connecting the macro- and microstrain responses in technical porous ceramics: modeling and experimental validations, Journal of Materials Science 46 (2011) 161-173.

[41] T.W. Darling, TenCate, J. A., Brown, D. W., Clausen, B., Vogel, S. C. Neutron diffraction study of the contribution of grain contacts to nonlinear stress-strain behavior, GEOPHYSICAL RESEARCH LETTERS 31 (2004) L16604.

[42] D.J. Tallman, M. Naguib, B. Anasori, M.W. Barsoum. Tensile creep of Ti2AlC in air in the temperature range 1000-1150 degrees C, Scripta Materialia 66 (2012) 805-808.

[43] F. Barcelo, S. Doriot, T. Cozzika, M. Le Flem, J.L. Bechade, M. Radovic, M.W. Barsoum. Electron-backscattered diffraction and transmission electron microscopy study of post-creep Ti3SiC2, Journal of Alloys and Compounds 488 (2009) 181-189.

[44] F.E. Hauser, C.D. Starr, L. Tietz, J.E. Dorn. Deformation mechanisms in polycrystalline aggregates of magnesium, Trans. Am. Soc. Metals. 47 (1955) 102-134.

[45] S. Basu, A. Zhou, M.W. Barsoum. On spherical nanoindentations, kinking nonlinear elasticity of mica single crystals and their geological implications, Journal of Structural Geology 31 (2009) 791-801. 
[46] M.W. Barsoum, A. Murugaiah, S.R. Kalidindi, T. Zhen, Y. Gogotsi. Kink bands, nonlinear elasticity and nanoindentations in graphite, Carbon 42 (2004) 1435-1445.

[47] N.J. Lane, S.I. Simak, A.S. Mikhaylushkin, I.A. Abrikosov, L. Hultman, M.W. Barsoum. Firstprinciples study of dislocations in hep metals through the investigation of the (\$11loverline $\{2\} 1 \$)$ twin boundary, Physical Review B 84 (2011) 184101.

[48] F.R.N. Nabarro. Theory of Crystal Dislocations Oxford University Press, Oxford, 1967. 

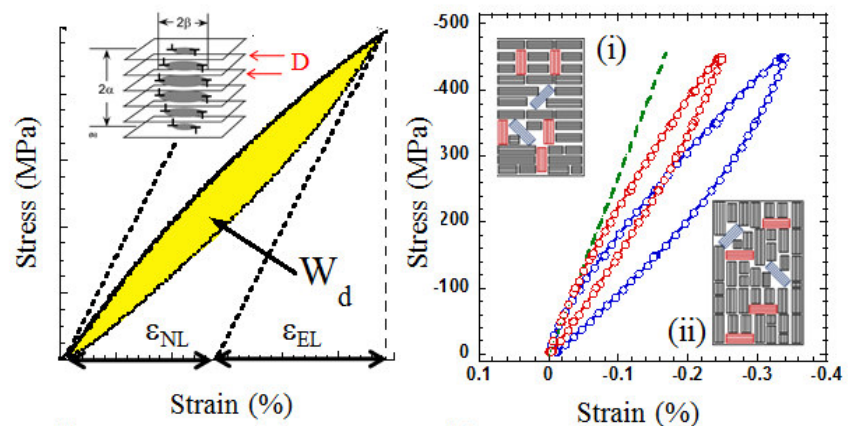

(a)

(b)

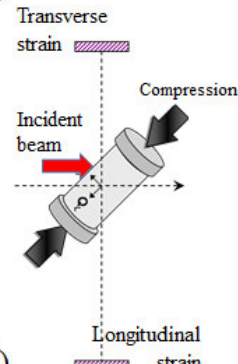

(c) strain

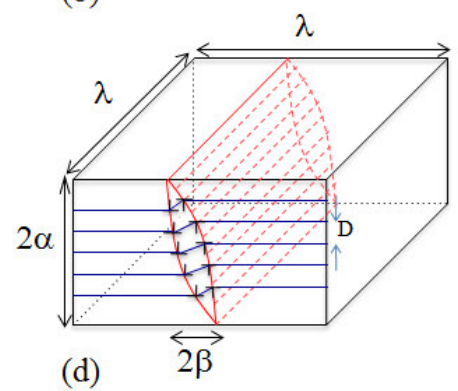

Figure 1: 


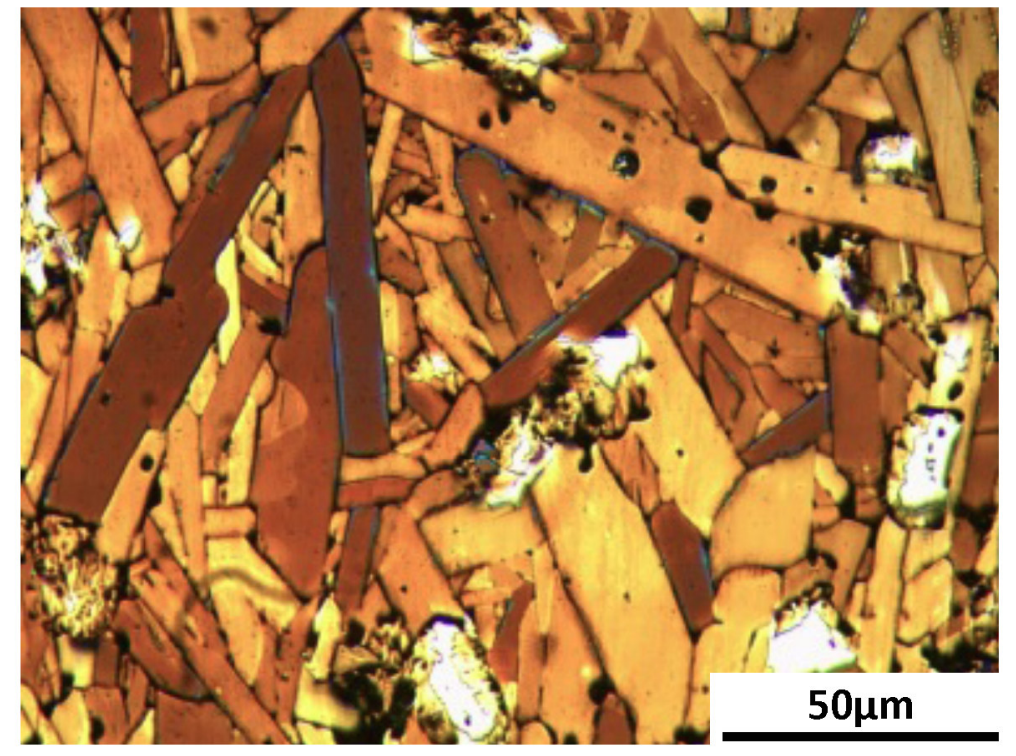

Figure 2 


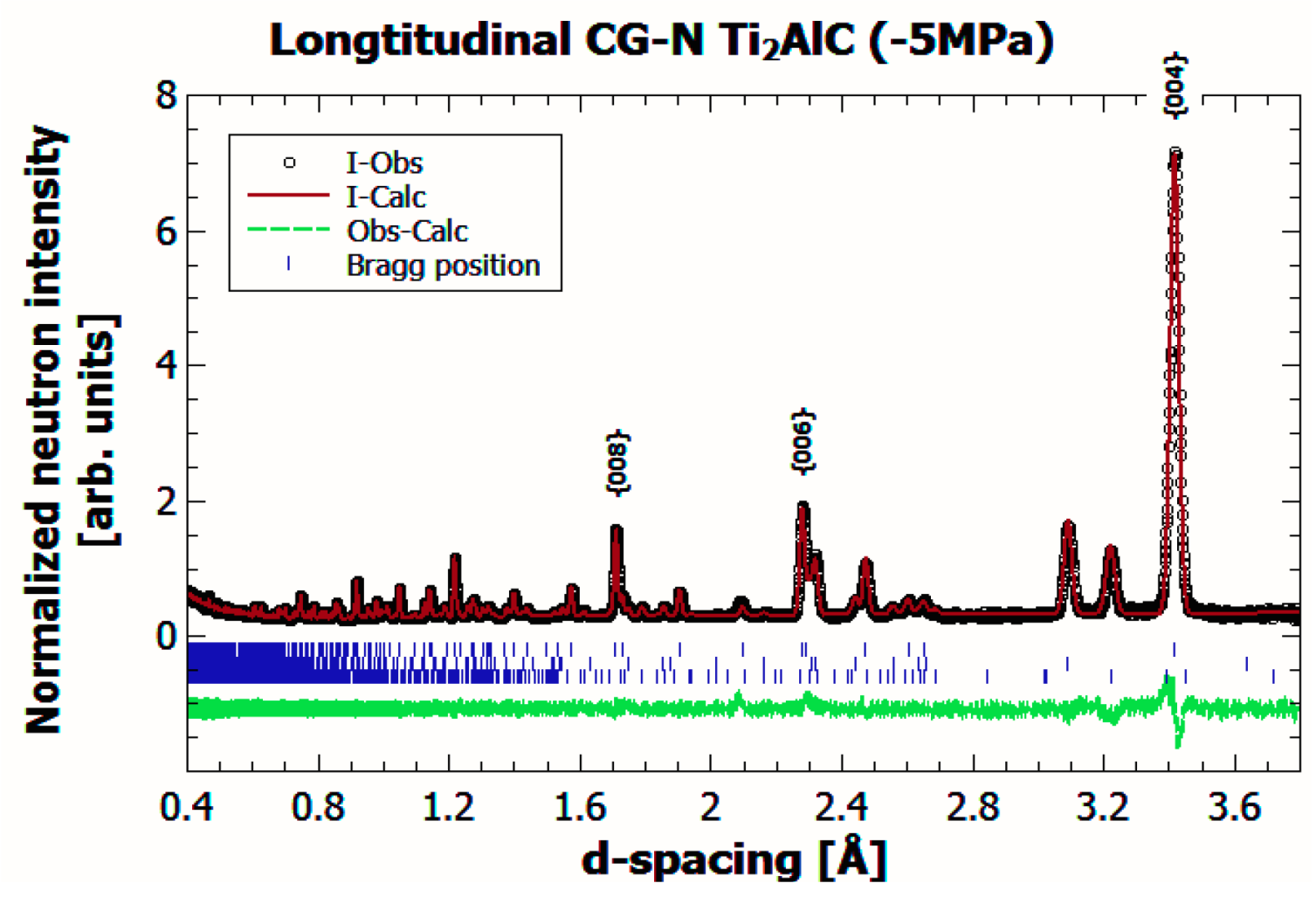

Figure 3 
Normal

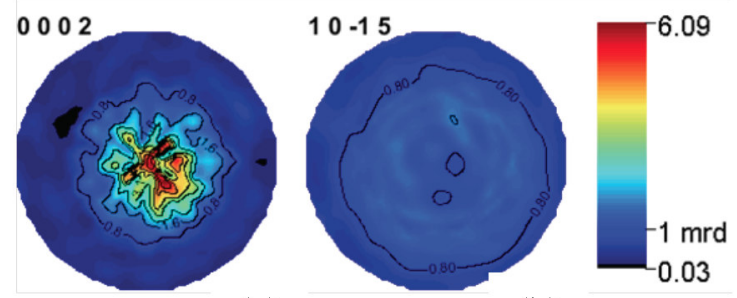

(a)

(b)

\section{Parallel}

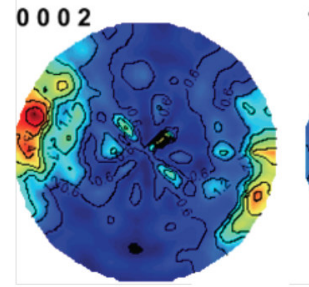

(c)

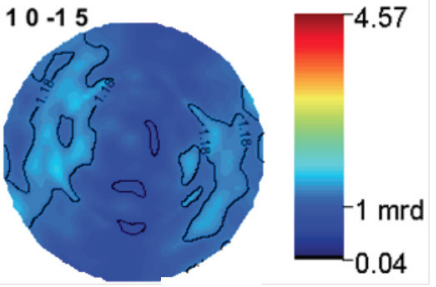

(d)

Figure 4 


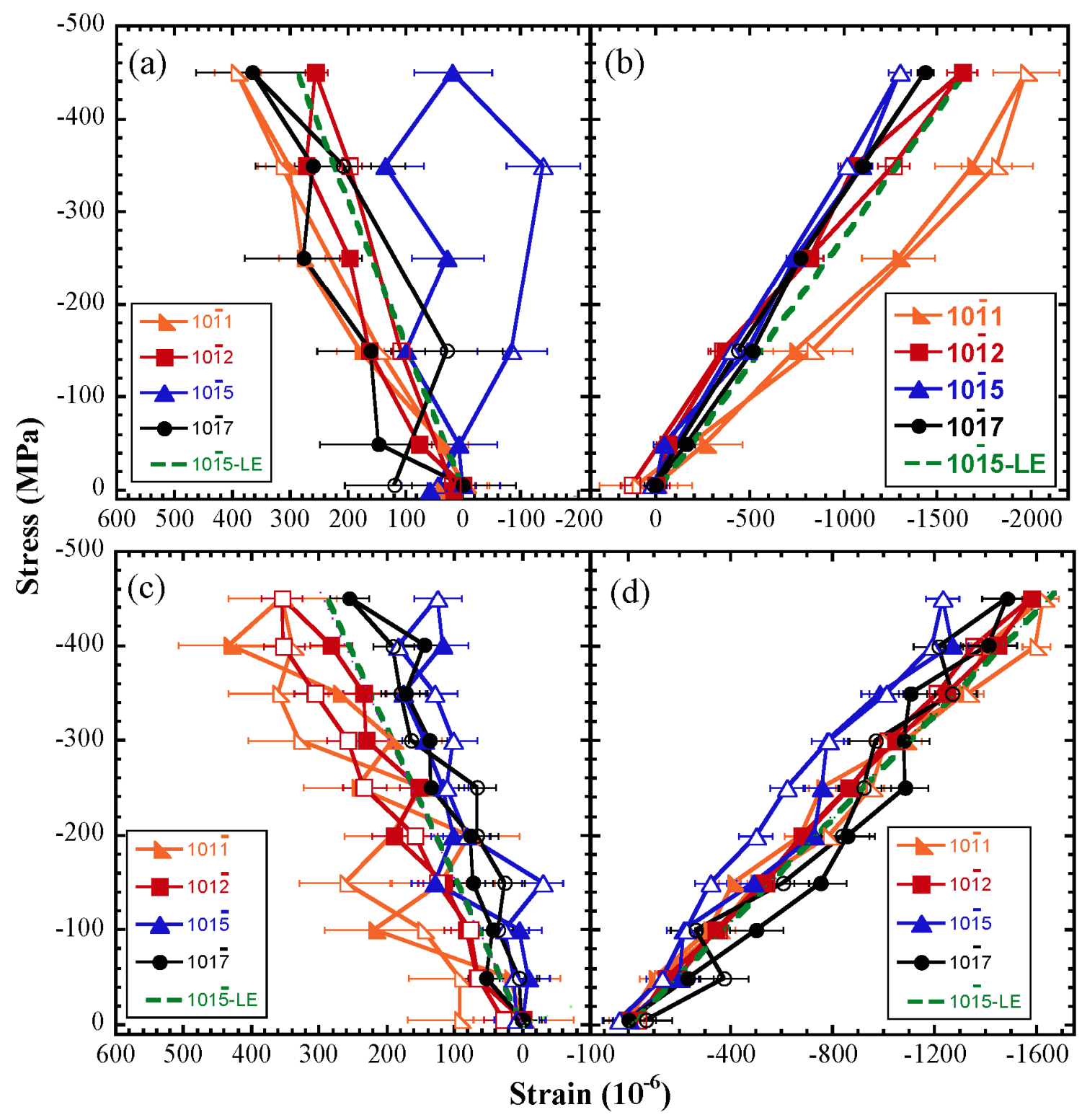

Figure 5 


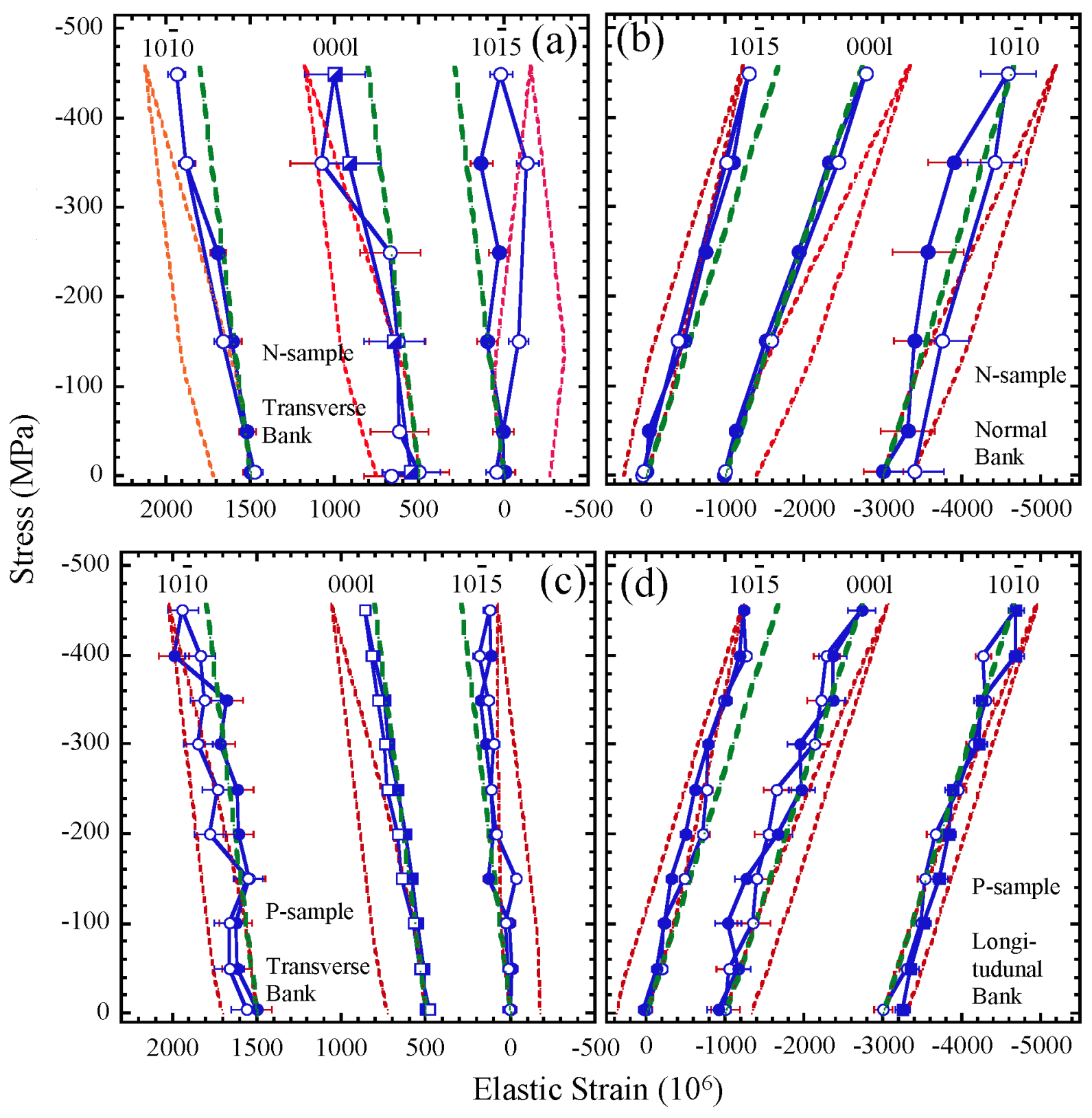

Figure 6 


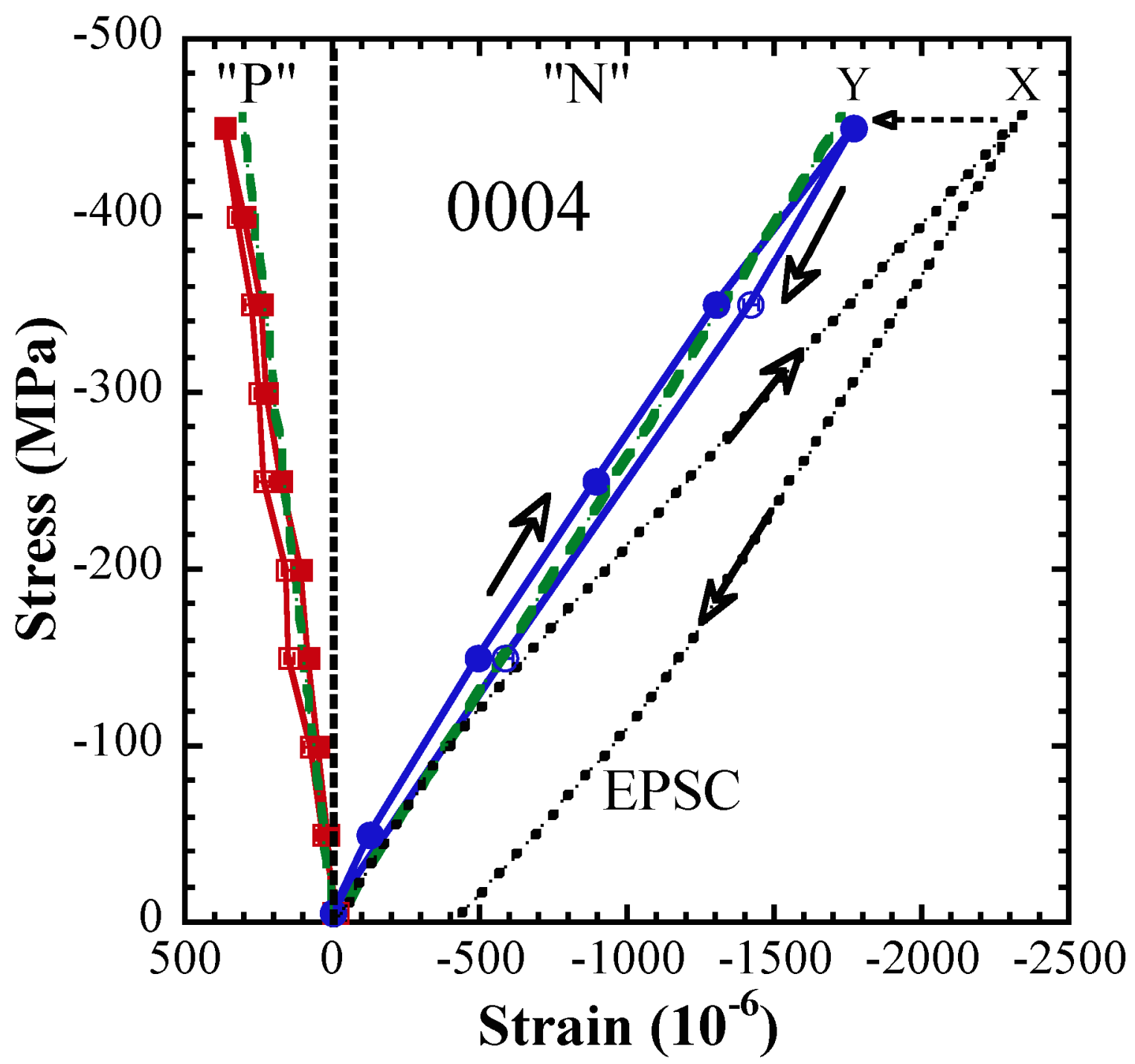

Figure 7 


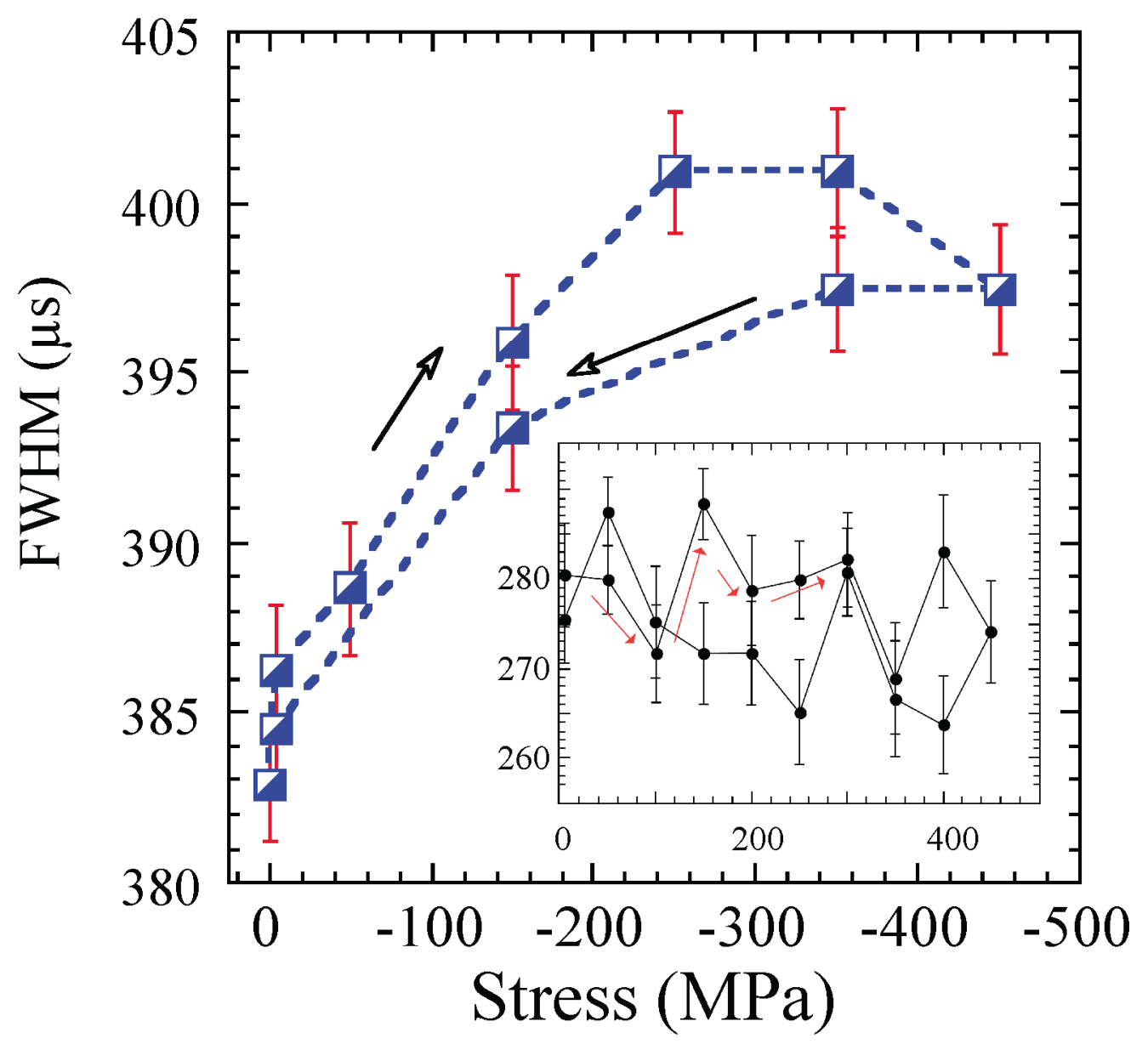

Figure 8 


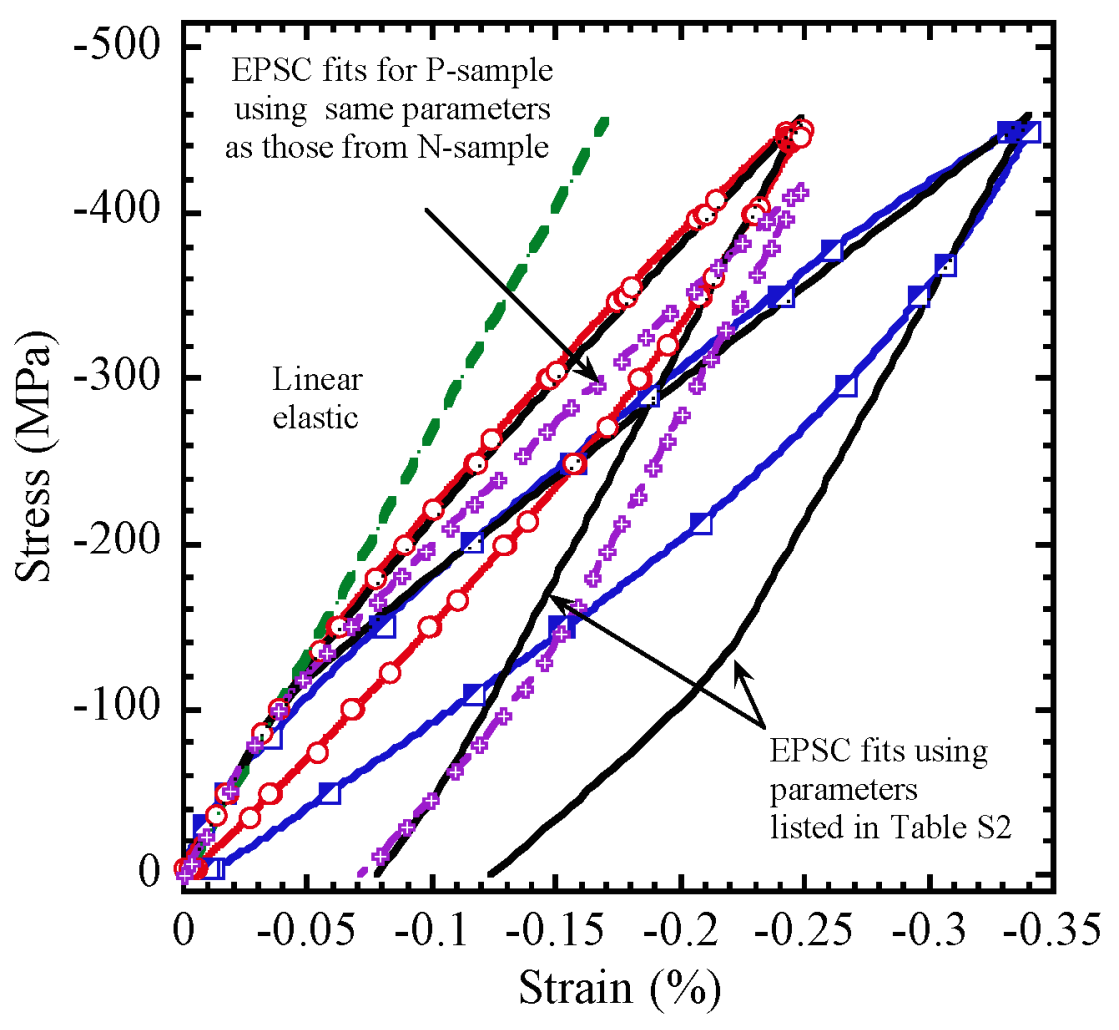

Figure 9 


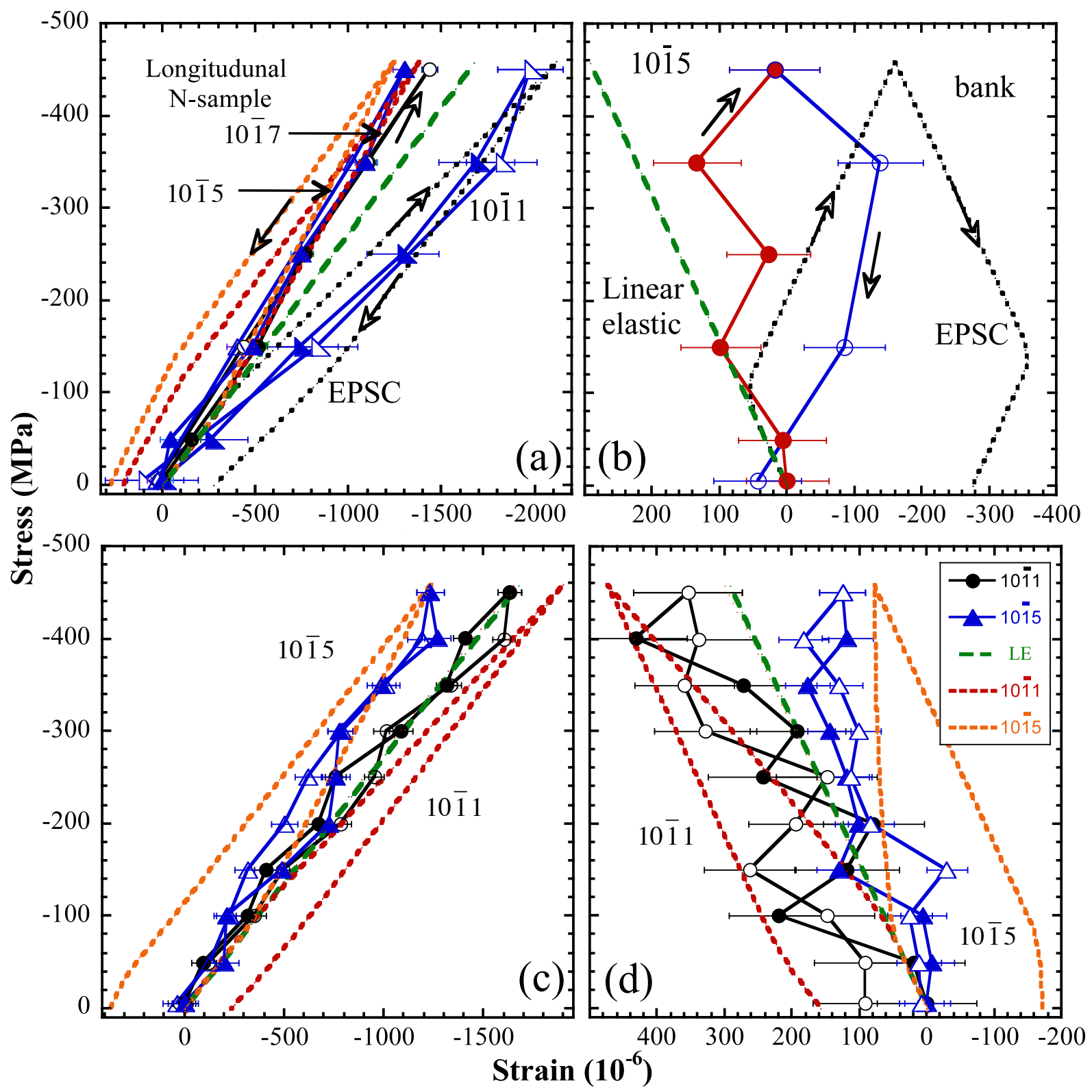

Figure 10: Stress-elastic strain response obtained from single peak fits of the ND results for a,b) of, 10-15, 10-17 and 10-11 reflections along, a) longitudinal and, b) transverse directions for the N-orientation. c,d) 10-15 and 10-11 reflections along, c) longitudinal and d) transverse directions for the P-orientation. Results obtained on loading are represented by solid symbols; those on unloading by open ones. The EPSC model results, assuming linear elastic behavior are depicted by dashed green lines; those for the 10-15 and 10-11 reflections, after activating basal slip are given by the orange and red dashed lines respectively. Both axes are reversed to render the plots similar to conventional tensile stress-strain curves. LE is an abbreviation for linear elastic. 


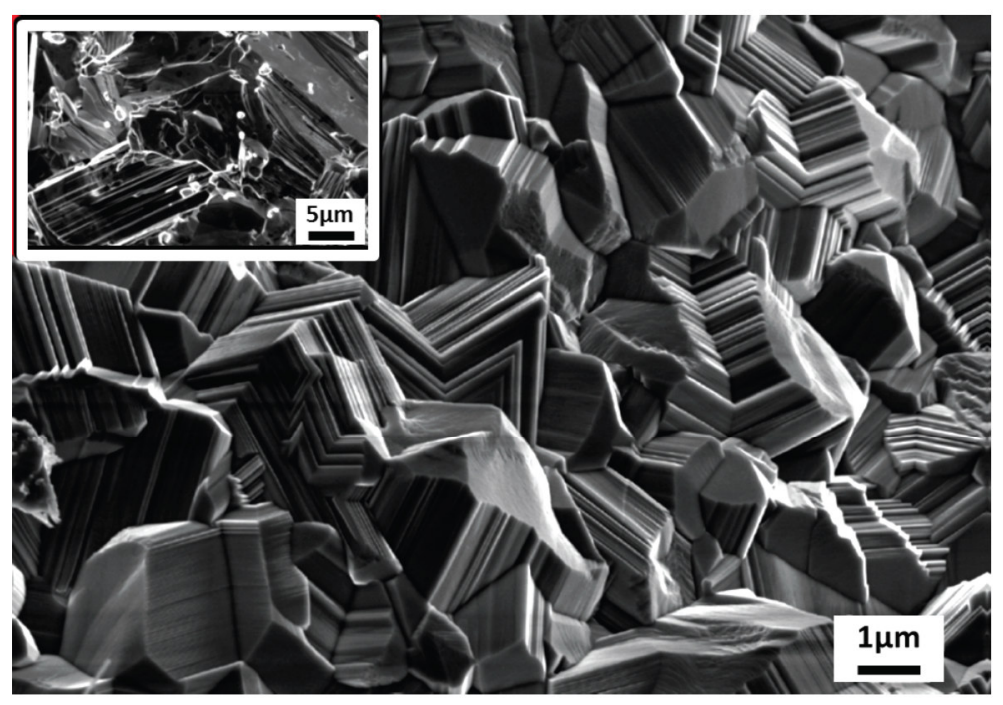

Figure 11 